\title{
Green function and Poisson kernel associated to root systems for annular regions
}

\author{
Chaabane REJEB*
}

\begin{abstract}
Let $\Delta_{k}$ be the Dunkl Laplacian relative to a fixed root system $\mathcal{R}$ in $\mathbb{R}^{d}, d \geq 2$, and to a nonnegative multiplicity function $k$ on $\mathcal{R}$. Our first purpose in this paper is to solve the $\Delta_{k}$-Dirichlet problem for annular regions. Secondly, we introduce and study the $\Delta_{k}$-Green function of the annulus and we prove that it can be expressed by means of $\Delta_{k}$-spherical harmonics. As applications, we obtain a Poisson-Jensen formula for $\Delta_{k}$-subharmonic functions and we study positive continuous solutions for a $\Delta_{k}$-semilinear problem.
\end{abstract}

MSC (2010) primary: 31B05, 35J08, 35J65; secondary: 31C45, 46F10, 47B39.

Key words: Dunkl-Laplace operator, Poisson kernel, Green function, Dirichlet problem, spherical harmonics, Newton kernel.

\section{Introduction}

Since the 90's, extensive studies have been carried out on analysis associated with Dunkl operators. These are commuting differential-difference operators on $\mathbb{R}^{d}$ introduced by $\mathrm{C}$. F. Dunkl (see [6]). The Dunkl analysis includes especially a generalization of the Fourier transform (called the Dunkl transform) and the Laplace operator known as the Dunkl Laplacian (and denoted by $\Delta_{k}$ ).

The Dunkl theory has many applications as well in mathematical physics and probability theory. In particular, it has been used in the study of the Calogero-Moser-Sutherland and other integrable systems (see $[4,10]$ ) and in the study of Markov processes generalizing Brownian motion (see [22]).

Recently, a special interest has been devoted to potential theory associated with the Dunkl Laplacian. The study focused on $\Delta_{k}$-harmonic functions (see $[2,11,12,17,19,20]$ ), on $\Delta_{k}$-Newton potential theory (including $\Delta_{k}$-subharmonic functions) (see [13]) and on $\Delta_{k^{-}}$ Riesz potentials of Radon measures (see [14]). More recently, by means of the $\Delta_{k}$-Newton kernel, the Green function of the open unit ball has been studied in [15]. Note that finding

\footnotetext{
${ }^{*}$ Université de Tunis El Manar, Faculté des Sciences de Tunis, Laboratoire d'Analyse Mathématques et Applications LR11ES11, 2092 El Manar I, Tunis, TUNISIA and Laboratoire de Mathématiques et Physique Théorique CNRS-UMR 7350, Université de Tours, Campus de Grandmont, 37200 Tours, FRANCE; Email: chaabane.rejeb@gmail.com
} 
$\Delta_{k}$-Green functions for other open sets is a rather difficult problem already in the case of the classical Laplace operator. The aim of this paper is to show that we can determine the $\Delta_{k}$-Green function for annular regions in $\mathbb{R}^{d}$ by using $\Delta_{k}$-spherical harmonics as a crucial tool.

Let us assume throughout the paper that $d \geq 2$. Let $A$ be the annulus

$$
\left.A:=\left\{x \in \mathbb{R}^{d}, \rho<\|x\|<1\right\} \quad \text { with } \rho \in\right] 0,1[.
$$

After giving some properties of the $\Delta_{k}$-Green function $G_{k, A}$ of $A$, we will use it to study the semilinear problem

$$
\begin{cases}\Delta_{k}\left(u \omega_{k}\right)=\phi(., u) \omega_{k}, & \text { in the sense of distributions } \\ u=f, & \text { on } \partial A,\end{cases}
$$

where $\omega_{k}$ is a precise weight function (see (2.6) for its expression).

More precisely, under some assumptions on the function $\phi$, we will show that if $f \in \mathcal{C}(\partial A)$ is nonnegative, this boundary problem has only and only one positive continuous solution on $A$ which satisfies (see Theorem 5.2)

$$
\forall x \in A, \quad u(x)+\int_{A} G_{k, A}(x, y) \phi(y, u(y)) \omega_{k}(y) d y=P_{k, A}[f](x) .
$$

Here $P_{k, A}[f]$ is the unique solution in $\mathcal{C}^{2}(A) \cap \mathcal{C}(\bar{A})$ of the boundary Dirichlet problem

$$
\begin{cases}\Delta_{k} u=0, & \text { on } A, \\ u=f, & \text { on } \partial A,\end{cases}
$$

that will be given explicitly in Section 3 .

This paper is organized as follows. In Section 2, we recall some basics from Dunkl theory that will be used throughout the paper. In Section 3, we give an explicit solution of the boundary Dirichlet problem for the annulus. The Green function $G_{k, A}$ will be introduced and studied in Section 4. Some applications will be given in the last Section. Precisely, we will obtain a Poisson-Jensen formula for $\Delta_{k}$-subharmonic functions in the annulus and we will study positive solutions of the above semilinear problem.

\section{Basics from Dunkl theory}

We start by recalling some useful facts in the Dunkl theory. Let $\mathcal{R}$ be a root system in the Euclidian space $\mathbb{R}^{d}$, in the sense that $\mathcal{R}$ is a finite set in $\mathbb{R}^{d} \backslash\{0\}$ such that for every $\alpha \in \mathcal{R}, \mathcal{R} \cap \mathbb{R} \alpha=\{ \pm \alpha\}$ and $\sigma_{\alpha}(\mathcal{R})=\mathcal{R}$ (where $\sigma_{\alpha}$ is the reflection w.r.t. the hyperplane $H_{\alpha}$ orthogonal to $\alpha$ ). The subgroup $W \subset O\left(\mathbb{R}^{d}\right)$ generated by the reflections $\sigma_{\alpha}, \alpha \in \mathcal{R}$, is called the Coxeter-Weyl group associated to $\mathcal{R}$. We refer to $([18])$ for more details on root systems and their Coxeter-Weyl groups.

Let $k$ be a fixed nonnegative multiplicity function on $\mathcal{R}$ (i.e. $k$ is $W$-invariant). For $\xi \in \mathbb{R}^{d}$, the $\xi$-directional Dunkl operator associated to $(W, k)$ is defined by

$$
D_{\xi} f(x):=\partial_{\xi} f(x)+\sum_{\alpha \in \mathcal{R}_{+}} k(\alpha)\langle\alpha, \xi\rangle \frac{f(x)-f\left(\sigma_{\alpha} \cdot x\right)}{\langle\alpha, x\rangle}, \quad f \in \mathcal{C}^{1}\left(\mathbb{R}^{d}\right),
$$


where $\partial_{\xi}$ is the usual $\xi$-directional partial derivative and $\mathcal{R}_{+}$is a positive subsystem. Let us denote by $\mathcal{P}\left(\mathbb{R}^{d}\right)$ (resp. $\mathcal{P}_{n}\left(\mathbb{R}^{d}\right)$ ) the space of polynomial functions on $\mathbb{R}^{d}$ (resp. the space of homogeneous polynomials of degree $n \in \mathbb{N}$ ).

It was shown that there is a unique linear isomorphism $V_{k}$ from $\mathcal{P}\left(\mathbb{R}^{d}\right)$ onto itself such that $V_{k}\left(\mathcal{P}_{n}\left(\mathbb{R}^{d}\right)\right)=\mathcal{P}_{n}\left(\mathbb{R}^{d}\right)$ for every $n \in \mathbb{N}, V_{k}(1)=1$ and

$$
\forall \xi \in \mathbb{R}^{d}, \quad D_{\xi} V_{k}=\partial_{\xi} V_{k} .
$$

The operator $V_{k}$ is known as the Dunkl intertwining operator (see [7, 8]). It has been extended to a topological isomorphism from $\mathcal{C}^{\infty}\left(\mathbb{R}^{d}\right)$ onto itself satisfying (2.1) (see [26]). Furthermore, according to [23], for each $x \in \mathbb{R}^{d}$, there is a compactly supported probability measure $\mu_{x}$ on $\mathbb{R}^{d}$ such that

$$
\forall f \in \mathcal{C}^{\infty}\left(\mathbb{R}^{d}\right), \quad V_{k}(f)(x)=\int_{\mathbb{R}^{d}} f(y) d \mu_{x}(y) .
$$

If $W \cdot x$ denotes the orbit of $x$ under the $W$-action and $C o(x)$ its convex hull, then

$$
\operatorname{supp} \mu_{x} \subset C o(x) \subset \bar{B}(0,\|x\|) .
$$

The Dunkl-Laplacian is defined as $\Delta_{k}=\sum_{j=1}^{d} D_{e_{j}}^{2}$, where $\left(e_{j}\right)_{1 \leq j \leq d}$ is the canonical basis of $\mathbb{R}^{d}$. It can be expressed as follows

$$
\Delta_{k} f(x)=\Delta f(x)+\sum_{\alpha \in R_{+}} k(\alpha)\left(2 \frac{\langle\nabla f(x), \alpha\rangle}{\langle\alpha, x\rangle}-\|\alpha\|^{2} \frac{f(x)-f\left(\sigma_{\alpha}(x)\right)}{\langle\alpha, x\rangle^{2}}\right), \quad f \in \mathcal{C}^{2}\left(\mathbb{R}^{d}\right),
$$

where $\Delta$ (resp. $\nabla$ ) is the usual Laplace (resp. gradient) operator (see $[6,8]$ ). Note that if $k$ is the zero function, the Dunkl Laplacian reduces to the classical one which is commutes with the action of $O\left(\mathbb{R}^{d}\right)$. For general $k \geq 0, \Delta_{k}$ commutes with the $W$-action (see [24]) i.e.

$$
\forall g \in W, \quad g \circ \Delta_{k}=\Delta_{k} \circ g .
$$

Let $L_{k}^{2}\left(S^{d-1}\right), d \geq 2$, be the Hilbert space endowed with the inner product

$$
\langle p, q\rangle_{k}:=\frac{1}{d_{k}} \int_{S^{d-1}} p(\xi) q(\xi) \omega_{k}(\xi) d \sigma(\xi) .
$$

We denote by $\|\cdot\|_{L_{k}^{2}\left(S^{d-1}\right)}$ the associated Euclidean norm. Here, $d \sigma$ is the surface measure on the unit sphere $S^{d-1}, \omega_{k}$ is the weight function given by

$$
\omega_{k}(x)=\prod_{\alpha \in \mathcal{R}_{+}}|\langle\alpha, x\rangle|^{2 k(\alpha)}
$$

and $d_{k}$ is the constant

$$
d_{k}=\int_{S^{d-1}} \omega_{k}(\xi) d \sigma(\xi) .
$$

The function $\omega_{k}$ is $W$-invariant and homogeneous of degree $2 \gamma:=2 \sum_{\alpha \in \mathcal{R}_{+}} k(\alpha)$. To simplify, we introduce the constant

$$
\lambda_{k}:=\frac{d}{2}+\gamma-1 \geq 0
$$


Let $\mathcal{H}_{\Delta_{k}, n}\left(\mathbb{R}^{d}\right):=\mathcal{P}_{n}\left(\mathbb{R}^{d}\right) \cap \operatorname{Ker} \Delta_{k}$ be the space of $\Delta_{k}$-harmonic polynomials, homogeneous of degree $n$ on $\mathbb{R}^{d}$. From [8], we know that if $n \neq m$, then $\mathcal{H}_{\Delta_{k}, n}\left(\mathbb{R}^{d}\right) \perp \mathcal{H}_{\Delta_{k}, m}\left(\mathbb{R}^{d}\right)$ in $L_{k}^{2}\left(S^{d-1}\right)$. Moreover, for every $n \in \mathbb{N}$, we have

$$
\mathcal{P}_{n}\left(\mathbb{R}^{d}\right)=\bigoplus_{j=0}^{\lfloor n / 2\rfloor}\|x\|^{2 j} \mathcal{H}_{\Delta_{k}, n-2 j}\left(\mathbb{R}^{d}\right) .
$$

The restriction to the sphere $S^{d-1}$ of an element of $\mathcal{H}_{\Delta_{k}, n}\left(\mathbb{R}^{d}\right)$ is called a $\Delta_{k}$-spherical harmonic of degree $n$. The space of $\Delta_{k}$-spherical harmonics will be denoted by $\mathcal{H}_{\Delta_{k}, n}\left(S^{d-1}\right)$. This space has a reproducing kernel $Z_{k, n}$ uniquely determined by the properties (see $[5,8]$ )

i) for each $x \in S^{d-1}, Z_{k, n}(x,.) \in \mathcal{H}_{\Delta_{k}, n}\left(S^{d-1}\right)$,

ii) for every $f \in \mathcal{H}_{\Delta_{k}, n}\left(S^{d-1}\right)$, we have

$$
f(x)=\left\langle f, Z_{k, n}(x, .)\right\rangle_{k}=\frac{1}{d_{k}} \int_{S^{d-1}} f(\xi) Z_{k, n}(x, \xi) \omega_{k}(\xi) d \sigma(\xi), \quad x \in S^{d-1} .
$$

From this formula, we can see that

$$
\forall g \in W, \quad \forall x, y \in S^{d-1}, \quad Z_{k, n}(g x, g y)=Z_{k, n}(x, y) .
$$

In classical case (i.e. $k=0$ ), $Z_{0, n}(x,$.$) is known as the zonal harmonic of degree n$ (see $[1,5])$. Note that if $\left\{Y_{j, n}, j=1, \ldots, h(n, d):=\operatorname{dim}_{\Delta_{k}, n}\left(\mathbb{R}^{d}\right)\right\}$ is a real-orthonormal basis of $\mathcal{H}_{\Delta_{k}, n}\left(S^{d-1}\right)$ in $L_{k}^{2}\left(S^{d-1}\right)$, then

$$
Z_{k, n}(x, y)=\sum_{j=1}^{h(n, d)} Y_{j, n}(x) Y_{j, n}(y) .
$$

By means of the Dunkl intertwining operator and Gegenbauer polynomials, $Z_{k, n}$ is given explicitly by (see [5], Theorem 7.2.6. or [27])

$$
\forall x, y \in S^{d-1}, \quad Z_{k, n}(x, y)=\frac{\left(n+\lambda_{k}\right)\left(2 \lambda_{k}\right)_{n}}{\lambda_{k} . n !} V_{k}\left(P_{n}^{\lambda_{k}}(\langle., y\rangle)\right)(x),
$$

where $\lambda_{k}$ is the constant given by $(2.8), P_{n}^{\mu}, \mu>-1 / 2$, is the normalized Gegenbauer polynomial (see [8] p. 17) defined by

$$
P_{n}^{\mu}(x):=\frac{(-1)^{n}}{2^{n}(\mu+1 / 2)_{n}}\left(1-x^{2}\right)^{1 / 2-\mu} \frac{d^{n}}{d x^{n}}\left(1-x^{2}\right)^{n+\mu-1 / 2},
$$

and $(x)_{n}:=x(x+1) \ldots(x+n-1)$ is the Pochhammer symbol.

At the end of this section, to simplify, when $k=0$ we will use the usual notations $L^{2}\left(S^{d-1}\right)$ for $L_{0}^{2}\left(S^{d-1}\right), \mathcal{H}_{\Delta, n}$ for $\mathcal{H}_{\Delta_{0}, n}, \omega_{d-1}:=d_{0}$ the surface area of $S^{d-1}$ and $Z_{n}:=Z_{0, n}$. 


\section{$3 \quad \Delta_{k}$-Dirichlet problem for the annulus}

In this section, by introducing a Poisson type kernel, we will solve the Dirichlet problem for the Dunkl Laplacian in annular regions

$$
A_{R_{1}, R_{2}}:=\left\{x \in \mathbb{R}^{d}: R_{1}<\|x\|<R_{2}\right\} .
$$

Note that from the homogeneity property of $\Delta_{k}$ :

$$
\delta_{r} \circ \Delta_{k}=r^{-2} \Delta_{k} \circ \delta_{r}, \quad \text { with } \quad \delta_{r}(f)(x):=f(r x),
$$

it suffices to do this for the annular region $A_{\rho, 1}$ with $\left.\rho \in\right] 0,1[$ fixed. In the sequel, to simplify, we will use the notation $A$ instead of $A_{\rho, 1}$.

Recall that the $\Delta_{k}$-Poisson kernel of the unit ball (see [8]) is given by

$$
P_{k}(x, y)=\sum_{n=0}^{+\infty} Z_{k, n}(x, y)=\int_{\mathbb{R}^{d}} \frac{1-\|x\|^{2}}{\left(1-\langle x, z\rangle+\|x\|^{2}\right)^{\frac{d}{2}+\gamma}} d \mu_{y}(z), \quad(x, y) \in B \times S^{d-1} .
$$

From [8], we know that

$$
\frac{1}{d_{k}} \int_{S^{d-1}} P_{k}(x, \xi) \omega_{k}(\xi) d \sigma(\xi)=1
$$

We start by two preliminary useful results. For each $n \in \mathbb{N}$, we see that the restriction of the Dunkl intertwining operator

$$
V_{k}: \mathcal{H}_{\Delta, n}\left(\mathbb{R}^{d}\right) \longrightarrow \mathcal{H}_{\Delta_{k}, n}\left(\mathbb{R}^{d}\right)
$$

is a linear isomorphism.

In the first result, we will estimate the matrix-norms of this operator and of its inverse where the space $\mathcal{H}_{\Delta, n}\left(\mathbb{R}^{d}\right)$ (resp. $\mathcal{H}_{\Delta_{k}, n}\left(\mathbb{R}^{d}\right)$ ) is endowed with the $L^{2}\left(S^{d-1}\right)$-norm (resp. the $L_{k}^{2}\left(S^{d-1}\right)$-norm). More precisely,

Proposition 3.1 Let $n$ be a nonnegative integer.

1. For every $f \in \mathcal{H}_{\Delta, n}\left(\mathbb{R}^{d}\right)$, we have

$$
\left\|V_{k}(f)\right\|_{L_{k}^{2}\left(S^{d-1}\right)} \leq \operatorname{dim} \mathcal{H}_{\Delta, n}\left(\mathbb{R}^{d}\right)\|f\|_{L^{2}\left(S^{d-1}\right)} .
$$

2. For every $f \in \mathcal{H}_{\Delta_{k}, n}\left(\mathbb{R}^{d}\right)$, we have

$$
\left\|V_{k}^{-1}(f)\right\|_{L^{2}\left(S^{d-1}\right)} \leq \frac{\left(\gamma+\frac{d}{2}\right)_{n} \omega_{d-1}}{\left(\frac{d}{2}\right)_{n}} \operatorname{dim} \mathcal{H}_{\Delta, n}\left(\mathbb{R}^{d}\right)\|f\|_{L_{k}^{2}\left(S^{d-1}\right)} .
$$


Proof:1) Let $f \in \mathcal{H}_{\Delta, n}\left(\mathbb{R}^{d}\right)$. After rewriting the reproducing formula (2.10) in the classical case (i.e. $k=0$ ), applying it to $f$ and using Fubini's theorem, we get

$$
V_{k}(f)(x)=\frac{1}{\omega_{d-1}} \int_{S^{d-1}} f(\xi) V_{k}\left[Z_{n}(., \xi)\right](x) d \sigma(\xi), \quad x \in \mathbb{R}^{d} .
$$

But, from [1], Proposition 5.27, we have

$$
\forall z, \xi \in S^{d-1}, \quad\left|Z_{n}(z, \xi)\right| \leq \operatorname{dim} \mathcal{H}_{\Delta, n}\left(\mathbb{R}^{d}\right)
$$

which implies that

$$
\forall(z, \xi) \in \mathbb{R}^{d} \times S^{d-1}, \quad\left|Z_{n}(z, \xi)\right| \leq\left(\operatorname{dim} \mathcal{H}_{\Delta, n}\left(\mathbb{R}^{d}\right)\right)\|z\|^{n} .
$$

Thus, using the relations (2.3), (3.5) and (3.6) and Cauchy-Schwarz inequality, we obtain

$$
\forall x \in \mathbb{R}^{d}, \quad\left|V_{k}(f)(x)\right| \leq \operatorname{dim} \mathcal{H}_{\Delta, n}\left(\mathbb{R}^{d}\right)\|f\|_{L^{2}\left(S^{d-1}\right)}\|x\|^{n} .
$$

This implies that

$$
\left\|V_{k}(f)\right\|_{L_{k}^{2}\left(S^{d-1}\right)} \leq \operatorname{dim} \mathcal{H}_{\Delta, n}\left(\mathbb{R}^{d}\right)\|f\|_{L^{2}\left(S^{d-1}\right)} .
$$

2) Let $f \in \mathcal{H}_{\Delta_{k}, n}\left(\mathbb{R}^{d}\right)$. Applying the classical case of the formula (2.10) with $f \leftarrow V_{k}^{-1}(f)$ and using (3.6) and Cauchy-Schwarz inequality, we deduce that

$$
\forall x \in \mathbb{R}^{d}, \quad\left|V_{k}^{-1}(f)(x)\right| \leq \operatorname{dim}_{\Delta, n}\left(\mathbb{R}^{d}\right)\left\|V_{k}^{-1}(f)\right\|_{L^{2}\left(S^{d-1}\right)}\|x\|^{n} .
$$

Now, using the following result (see [8], Proposition 5.2.8): for $p \in \mathcal{P}_{n}\left(\mathbb{R}^{d}\right)$ and $q \in$ $\mathcal{H}_{\Delta, n}\left(\mathbb{R}^{d}\right)$, then

$$
\frac{1}{\omega_{d-1}} \int_{S^{d-1}} p(\xi) q(\xi) d \sigma(\xi)=\frac{\left(\gamma+\frac{d}{2}\right)_{n} \omega_{d-1}}{\left(\frac{d}{2}\right)_{n} d_{k}} \int_{S^{d-1}} p(\xi) V_{k}(q)(\xi) \omega_{k}(\xi) d \sigma(\xi)
$$

with $p=q=V_{k}^{-1}(f)$, we obtain

$$
\begin{aligned}
\left\|V_{k}^{-1}(f)\right\|_{L^{2}\left(S^{d-1}\right)}^{2} & \leq \frac{\left(\gamma+\frac{d}{2}\right)_{n} \omega_{d-1}}{\left(\frac{d}{2}\right)_{n} d_{k}} \int_{S^{d-1}}\left|V_{k}^{-1}(f)(\xi) f(\xi)\right| \omega_{k}(\xi) d \sigma(\xi) \\
& \leq \frac{\left(\gamma+\frac{d}{2}\right)_{n} \omega_{d-1}}{\left(\frac{d}{2}\right)_{n} d_{k}} \operatorname{dim}_{\Delta, n}\left(\mathbb{R}^{d}\right)\left\|V_{k}^{-1}(f)\right\|_{L^{2}\left(S^{d-1}\right)} \int_{S^{d-1}}|f(\xi)| \omega_{k}(\xi) d \sigma(\xi) \\
& \leq \frac{\left(\gamma+\frac{d}{2}\right)_{n} \omega_{d-1}}{\left(\frac{d}{2}\right)_{n}} \operatorname{dim\mathcal {H}}_{\Delta, n}\left(\mathbb{R}^{d}\right)\left\|V_{k}^{-1}(f)\right\|_{L^{2}\left(S^{d-1}\right)}\|f\|_{L_{k}^{2}\left(S^{d-1}\right)} .
\end{aligned}
$$

This proves the desired relation.

Corollary 3.1 The following inequality holds:

$$
\forall x, y \in S^{d-1}, \quad\left|Z_{k, n}(x, y)\right| \leq\left(\frac{\left(\gamma+\frac{d}{2}\right)_{n} \omega_{d-1}}{\left(\frac{d}{2}\right)_{n}}\right)^{2}\left(\operatorname{dim} \mathcal{H}_{\Delta, n}\left(\mathbb{R}^{d}\right)\right)^{5} .
$$


Proof: Let $\left\{Y_{j, n}, j=1, \ldots, h(n, d)=\operatorname{dim}_{\Delta_{k}, n}\left(\mathbb{R}^{d}\right)\right\}$, is a real-orthonormal basis of $\mathcal{H}_{\Delta_{k}, n}\left(\mathbb{R}^{d}\right)$ in $L_{k}^{2}\left(S^{d-1}\right)$. Using (3.7) with $f=V_{k}^{-1}\left(Y_{j, n}\right)$ and (3.4), we deduce that

$$
\begin{aligned}
\forall x \in S^{d-1},\left|Y_{j, n}(x)\right| & \leq \operatorname{dim} \mathcal{H}_{\Delta, n}\left(\mathbb{R}^{d}\right)\left\|V_{k}^{-1}\left(Y_{j, n}\right)\right\|_{L^{2}\left(S^{d-1}\right)} \\
& \leq \frac{\left(\gamma+\frac{d}{2}\right)_{n} \omega_{d-1}}{\left(\frac{d}{2}\right)_{n}}\left(\operatorname{dim} \mathcal{H}_{\Delta, n}\left(\mathbb{R}^{d}\right)\right)^{2} .
\end{aligned}
$$

Consequently, we obtain the result from (2.12).

Following the classical case $k=0$ (see [1]), we define the kernel $\mathbf{P}_{k, 1}(.,$.$) on A \times S^{d-1}$ by

$$
\mathbf{P}_{k, 1}(x, \xi):=\sum_{n=0}^{+\infty} a_{k, n}(x) Z_{k, n}(x, \xi), \quad \text { with } \quad a_{k, n}(x)=\frac{1-\left(\frac{\|x\|}{\rho}\right)^{-2 \lambda_{k}-2 n}}{1-\rho^{2 \lambda_{k}+2 n}} .
$$

Proposition 3.2 The kernel $\mathbf{P}_{k, 1}$ satisfies the following properties

i) For each $\xi \in S^{d-1}, \mathbf{P}_{k, 1}(., \xi)$ is a $\Delta_{k}$-harmonic function on $A$ and $\mathbf{P}_{k, 1}(., \xi)=0$ on $S(0, \rho)$.

ii) For every $x \in A$ and $\xi \in S^{d-1}$,

$$
0 \leq \mathbf{P}_{k, 1}(x, \xi) \leq P_{k}(x, \xi)
$$

iii) Let $x \in A$ and $\xi \in S^{d-1}$ fixed. Then

$$
\forall g \in W, \quad \mathbf{P}_{k, 1}(g x, g \xi)=\mathbf{P}_{k, 1}(x, \xi) .
$$

Proof: i) Clearly $\mathbf{P}_{k, 1}(., \xi)=0$ on $S(0, \rho)$. On the other hand, for any $(x, \xi) \in A \times S^{d-1}$ we can write

$$
a_{k, n}(x) Z_{k, n}(x, \xi)=c_{1, n} Z_{k, n}(x, \xi)-c_{2, n} K_{k}\left[Z_{k, n}(., \xi)\right](x),
$$

where $c_{1, n}, c_{2, n}$ are two nonnegative constants and $K_{k}$ is the $\Delta_{k}$-Kelvin transform (see [9]) given by

$$
K_{k}[f](x)=\|x\|^{-2 \lambda_{k}} f\left(x /\|x\|^{2}\right)=\|x\|^{2-2 \gamma-d} f\left(x /\|x\|^{2}\right)
$$

and $f$ is a function defined on $\mathbb{R}^{d} \backslash\{0\}$. As the $\Delta_{k}$-Kelvin transform preserves the $\Delta_{k^{-}}$ harmonic functions on $\mathbb{R}^{d} \backslash\{0\}$ (see [9]), we deduce that the function $x \mapsto a_{k, n}(x) Z_{k, n}(x, \xi)$ is $\Delta_{k}$-harmonic on $A$.

According to [8] (see also [1] and [5]), we know that

$$
\operatorname{dim} \mathcal{H}_{\Delta, n}\left(\mathbb{R}^{d}\right)=\operatorname{dim}_{\Delta_{k}, n}\left(\mathbb{R}^{d}\right)=\left(\begin{array}{c}
n+d-1 \\
n
\end{array}\right)-\left(\begin{array}{c}
n+d-3 \\
n-2
\end{array}\right) .
$$

Hence, we have

$$
\lim _{n \rightarrow+\infty} n^{2-d} \operatorname{dim}_{\Delta, n}\left(\mathbb{R}^{d}\right)=\frac{2}{(d-2) !}
$$


Moreover, we have

$$
\lim _{n \rightarrow+\infty} n^{-\gamma} \frac{\left(\gamma+\frac{d}{2}\right)_{n}}{\left(\frac{d}{2}\right)_{n}}=\lim _{n \rightarrow+\infty} n^{-\gamma} \frac{\Gamma(d / 2)}{\Gamma(\gamma+d / 2)} \frac{\Gamma(d / 2+\gamma+n)}{\Gamma(d / 2+n)}=\frac{\Gamma(d / 2)}{\Gamma(\gamma+d / 2)} .
$$

Consequently, from (3.8), there exists $C=C(d, \gamma)>0$ such that

$$
\forall x \in \mathbb{R}^{d}, \quad \forall y \in S^{d-1}, \quad\left|Z_{k, n}(x, y)\right| \leq C n^{5 d+2 \gamma-10}\|x\|^{n} .
$$

This inequality as well as the fact that $0 \leq a_{k, n}(x)<1$ imply that the series

$$
\sum_{n \geq 0} a_{k, n}(x) Z_{k, n}(x, \xi)
$$

converges uniformly on $\bar{A}_{\rho, R} \times S^{d-1}$ for every $\left.R \in\right] \rho, 1$ [. Then , by Corollary 3.3 in [11], the function $\mathbf{P}_{k, 1}(., \xi)$ is $\Delta_{k}$-harmonic on $A$.

ii) For $\varepsilon>0$ small enough and $\xi \in S^{d-1}$, consider the function

$$
h_{\varepsilon}(x):=\sum_{n \geq 0} a_{k, n}(x) Z_{k, n}((1-\varepsilon) x, \xi) .
$$

As above, from the inequality (3.13) and the homogeneity of $Z_{k, n}(., \xi)$, we see that $h_{\varepsilon}$ defines a $\Delta_{k}$-harmonic function in the annular region $A_{\rho, R}$ with $R=(1-\varepsilon)^{-1}$. Furthermore, $h_{\varepsilon}=0$ on $S(0, \rho)$ and if $x \in S^{d-1}$, then

$$
h_{\varepsilon}(x)=\sum_{n \geq 0} Z_{k, n}((1-\varepsilon) x, \xi)=P_{k}((1-\varepsilon) x, \xi) .
$$

where $P_{k}$ is the $\Delta_{k}$-Poisson kernel of the unit ball (see [8]). In particular, $h_{\varepsilon} \geq 0$ on $S^{d-1}$. Consequently, by the weak minimum principle for $\Delta_{k}$-harmonic functions (see [11] or [21]), we deduce that

$$
\forall x \in A, \quad h_{\varepsilon}(x) \geq 0 .
$$

On the other hand, for each fixed $(x, \xi)$ in $A \times S^{d-1}$, we have

$$
\begin{aligned}
\left|\mathbf{P}_{k, 1}(x, \xi)-h_{\varepsilon}(x)\right| & \leq \sum_{n \geq 1}\left(1-(1-\varepsilon)^{n}\right) a_{k, n}(x)\left|Z_{k, n}(x, \xi)\right| \\
& \leq C \sum_{n \geq 1}\left(1-(1-\varepsilon)^{n}\right) n^{5 d+2 \gamma-10}\|x\|^{n} .
\end{aligned}
$$

Hence, by the monotone convergence theorem we have $\mathbf{P}_{k, 1}(x, \xi)=\lim _{\varepsilon \rightarrow 0} h_{\varepsilon}(x)$. Finally, we obtain $\mathbf{P}_{k, 1} \geq 0$ on $A \times S^{d-1}$.

- For $\xi \in S^{d-1}$ fixed, the function $x \mapsto P_{k}((1-\varepsilon) x, \xi)-h_{\varepsilon}(x)$ is $\Delta_{k}$-harmonic on $A$. Moreover, since $P_{k}$ is a nonnegative kernel, we have

$$
\forall x \in S(0, \rho), \quad P_{k}((1-\varepsilon) x, \xi)-h_{\varepsilon}(x)=P_{k}((1-\varepsilon) x, \xi) \geq 0 .
$$

By (3.14), $x \mapsto P_{k}((1-\varepsilon) x, \xi)-h_{\varepsilon}(x)$ is the zero function on $S^{d-1}$. So, the weak maximum principle implies that

$$
\forall x \in A, \quad P_{k}((1-\varepsilon) x, \xi) \geq h_{\varepsilon}(x) .
$$

Letting $\varepsilon \longrightarrow 0$, we obtain $P_{k}(., \xi) \geq \mathbf{P}_{k, 1}(., \xi)$ on $A$.

iii) The result follows immediately from (2.11). 
Proposition 3.3 Let $f$ be a continuous function on $S^{d-1}$. Then the function

$$
\mathbf{P}_{k, 1}[f](x)=\frac{1}{d_{k}} \int_{S^{d-1}} \mathbf{P}_{k, 1}(x, \xi) f(\xi) \omega_{k}(\xi) d \sigma(\xi)
$$

is the unique solution in $\mathcal{C}^{2}(A) \cap \mathcal{C}(\bar{A})$ of the boundary Dirichlet problem

$$
\begin{cases}\Delta_{k} u=0, & \text { on } A \\ u=f, & \text { on } S^{d-1} \\ u=0, & \text { on } S(0, \rho) .\end{cases}
$$

Proof: The uniqueness follows from the weak maximum principle for $\Delta_{k}$-harmonic functions (see [11] or [21]). The inequality (3.13) allowed us to write for any $x \in A$ that

$$
\mathbf{P}_{k, 1}[f](x)=\sum_{n=0}^{+\infty} u_{n}(x), \quad \text { with } \quad u_{n}(x)=\frac{a_{k, n}(x)}{d_{k}} \int_{S^{d-1}} Z_{k, n}(x, \xi) f(\xi) \omega_{k}(\xi) d \sigma(\xi) .
$$

By differentiation theorem under integral sign, the functions $u_{n}$ are $\Delta_{k}$-harmonic on $A$. Moreover, by (3.13) we have

$$
\forall n, \quad\left|u_{n}(x)\right| \leq C\|f\|_{\infty} n^{5 d+2 \gamma-10}\|x\|^{n} .
$$

This proves that the series $\sum_{n \geq 0} u_{n}$ converges uniformly on each closed annular region $\bar{A}_{\rho, R}$ whenever $\left.R \in\right] \rho, 1\left[\right.$. Then, we conclude that $\mathbf{P}_{k, 1}[f]$ is $\Delta_{k}$-harmonic on $A$.

On the other hand, it is easy to see that $\mathbf{P}_{k, 1}[f]=0$ on $S(0, \rho)$.

It remains to prove that for every $\xi \in S^{d-1}, \lim _{x \rightarrow \xi} \mathbf{P}_{k, 1}[f](x)=f(\xi)$.

- If $f \in \mathcal{H}_{\Delta_{k}, m}\left(\mathbb{R}^{d}\right)$, then $u_{n}=0$ if $n \neq m$ and $u_{m}(x)=a_{k, m}(x) f(x)=\mathbf{P}_{k, 1}[f](x)$. Therefore, $\mathbf{P}_{k, 1}[f]=f$ on $S^{d-1}$.

- If $f \in \mathcal{P}_{m}\left(\mathbb{R}^{d}\right)$, then by $(2.9)$, there exist $f_{1}, \ldots, f_{m}$, with $f_{j} \in \mathcal{H}_{\Delta_{k}, n-2 j}\left(\mathbb{R}^{d}\right)$ such that

$$
f(x)=\sum_{j=0}^{[m / 2]}\|x\|^{2 j} f_{j}(x)
$$

This implies that $\mathbf{P}_{k, 1}[f]=f$ on $S^{d-1}$.

- If $f$ is an arbitrary polynomial function, the result also holds.

- Suppose that $f$ is a continuous function on $S^{d-1}$ and let $p$ be a polynomial function.

By (3.10) and (3.2) we have

$$
\begin{aligned}
\left|\mathbf{P}_{k, 1}[f](x)-f(x)\right| & \leq\left|\mathbf{P}_{k, 1}[f](x)-\mathbf{P}_{k, 1}[p](x)\right|+\left|\mathbf{P}_{k, 1}[p](x)-p(x)\right|+|p(x)-f(x)| \\
& \leq 2\|f-p\|_{\infty}+\left|\mathbf{P}_{k, 1}[p](x)-p(x)\right| .
\end{aligned}
$$

This inequality as well as the Stone-Weierstrass theorem show that $\lim _{x \rightarrow \xi} \mathbf{P}_{k, 1}[f](x)=$ $f(\xi)$ for every $\xi \in S^{d-1}$. This completes the proof.

Now, for $x \in A$ and $\xi \in S^{d-1}$, consider the functions

$$
b_{k, n}(x)=\|x\|^{-n}\left(\frac{\|x\|}{\rho}\right)^{-2 \lambda_{k}-n} \frac{1-\|x\|^{2 \lambda_{k}+2 n}}{1-\rho^{2 \lambda_{k}+2 n}}=\rho^{-n}\left(1-a_{k, n}(x)\right)
$$


and

$$
\mathbf{P}_{k, 2}(x, \xi):=\sum_{n=0}^{+\infty} b_{k, n}(x) Z_{k, n}(x, \xi) .
$$

By means of the Poisson kernel of the unit ball, we can write

$$
\mathbf{P}_{k, 2}(x, \rho \xi)=P_{k}(x, \xi)-\mathbf{P}_{k, 1}(x, \xi) .
$$

This relation as well as the properties of $P_{k}$ and $\mathbf{P}_{k, 1}$ prove that $\mathbf{P}_{k, 2}(., \rho \xi)$ is a nonnegative $\Delta_{k}$-harmonic function on $A$ with $\mathbf{P}_{k, 2}(., \rho \xi)=0$ on $S^{d-1}$.

Let $f$ be a continuous function on $S(0, \rho)$ and define the function

$$
\mathbf{P}_{k, 2}[f](x)=\frac{1}{d_{k}} \int_{S^{d-1}} \mathbf{P}_{k, 2}(x, \rho \xi) f(\rho \xi) \omega_{k}(\xi) d \sigma(\xi), \quad x \in A .
$$

Using (3.17), we can write

$$
\begin{aligned}
\mathbf{P}_{k, 2}[f](x) & =\frac{1}{d_{k}} \int_{S^{d-1}}\left(P_{k}(x, \xi)-\mathbf{P}_{k, 1}(x, \xi)\right) f(\rho \xi) \omega_{k}(\xi) d \sigma(\xi) \\
& =P_{k}\left[\delta_{\rho} . f\right](x)-\mathbf{P}_{k, 1}\left[\delta_{\rho} . f\right](x) .
\end{aligned}
$$

Here, $P_{k}[\phi]$ denotes the Poisson integral of $\phi$ and $\delta_{\rho} . f(x)=f(\rho x)$.

Then, using Proposition 3.3 and theorem A in [19], we obtain immediately the following result:

Proposition 3.4 Let $f$ be a continuous function on $S(0, \rho)$. Then $\mathbf{P}_{k, 2}[f]$ is the unique solution in $\mathcal{C}^{2}(A) \cap \mathcal{C}(\bar{A})$ of the boundary Dirichlet problem

$$
\begin{cases}\Delta_{k} u=0, & \text { on } A ; \\ u=0, & \text { on } S^{d-1} \\ u=f, & \text { on } S(0, \rho) .\end{cases}
$$

Definition 3.1 Let $f$ be a continuous function on $\partial A$. We define the $\Delta_{k}$-Poisson integral of $f$ for the annulus $A$ by

$$
P_{k, A}[f](x):=\mathbf{P}_{k, 1}[f](x)+\mathbf{P}_{k, 2}[f](x)
$$

Remark 3.1 1. We can see that $P_{k, A}[1]=1$.

2. Using (3.11) and a similar relation for the kernel $\mathbf{P}_{k, 2}$, we obtain

$$
g \cdot P_{k, A}[f]=P_{k, A}[g . f], \text { with } \quad g \cdot f(x):=f\left(g^{-1} x\right) .
$$

From Propositions 3.3 and 3.4, we deduce the following main result:

Theorem 3.1 Let $f \in \mathcal{C}(\partial A)$. Then the function $P_{k, A}[f]$ is the unique solution in $\mathcal{C}^{2}(A) \cap$ $\mathcal{C}(\bar{A})$ of the boundary Dirichlet problem

$$
\begin{cases}\Delta_{k} u=0, & \text { on } A ; \\ u=f & \text { on } \partial A .\end{cases}
$$


From this theorem and the weak maximum principle for $\Delta_{k}$-harmonic function (see [11]), we obtain the following result:

Corollary 3.2 Let $h$ be a $\Delta_{k}$-harmonic function on $A$ and continuous on $\bar{A}$. Then,

$$
\forall x \in A, \quad h(x)=P_{k, A}[h](x) .
$$

\section{$4 \quad \Delta_{k}$-Green function of the annulus}

Our aim in this section is to introduce and study the Green function of the annular region $A=\left\{x \in \mathbb{R}^{d}, \rho<\|x\|<1\right\}$ for the Dunkl-Laplace operator. In the sequel, we will assume that $d+2 \gamma>2$ i.e. $\lambda_{k}>0$ with $\lambda_{k}$ the constant (2.8).

Let us first recall that the $\Delta_{k}$-Newton kernel, introduced in [13], is given by

$$
N_{k}(x, y):=\int_{0}^{+\infty} p_{k}(t, x, y) d t
$$

with $p_{k}$ the Dunkl heat kernel (see $[21,24]$ )

$$
p_{k}(t, x, y)=\frac{1}{(2 t)^{d / 2+\gamma} c_{k}} \int_{\mathbb{R}^{d}} e^{-\left(\|x\|^{2}+\|y\|^{2}-2\langle x, z\rangle\right) / 4 t} d \mu_{y}(z)
$$

and $c_{k}$ the Macdonald-Mehta constant given by

$$
c_{k}:=\int_{\mathbb{R}^{d}} \exp \left(-\frac{\|x\|^{2}}{2}\right) \omega_{k}(x) d x .
$$

According to ([13]), the positive and symmetric kernel $N_{k}$ takes the following form

$$
N_{k}(x, y)=\frac{1}{2 d_{k} \lambda_{k}} \int_{\mathbb{R}^{d}}\left(\|x\|^{2}+\|y\|^{2}-2\langle x, z\rangle\right)^{-\lambda_{k}} d \mu_{y}(z) .
$$

Note that if $y=0, \mu_{y}=\delta_{0}$ (with $\delta_{x_{0}}$ the Dirac measure at $x_{0} \in \mathbb{R}^{d}$ ) and then

$$
N_{k}(x, 0)=\frac{1}{2 d_{k} \lambda_{k}}\|x\|^{-2 \lambda_{k}} .
$$

In addition, for each fixed $x \in \mathbb{R}^{d}$, the function $N_{k}(x,$.$) is \Delta_{k}$-harmonic and of class $C^{\infty}$ on $\mathbb{R}^{d} \backslash W . x$ (where $W . x$ is the $W$-orbit of $x$ ), $\Delta_{k}$-superharmonic (see below for precise definition) on whole $\mathbb{R}^{d}$ and satisfies

$$
-\Delta_{k}\left[N_{k}(x, .) \omega_{k}\right]=\delta_{x}, \quad \text { in } \quad \mathcal{D}^{\prime}\left(\mathbb{R}^{d}\right)
$$

where

-for $\Omega \subset \mathbb{R}^{d}$ a $W$-invariant open set, $\mathcal{D}(\Omega)$ and $\mathcal{D}^{\prime}(\Omega)$ denote respectively the space of $C^{\infty}$-functions on $\Omega$ with compact support and the space of Schwartz distributions on $\Omega$. -for $f \in L_{l o c}^{1}\left(\Omega, \omega_{k}(x) d x\right), \Delta_{k}\left(f \omega_{k}\right)$ is the Schwartz distribution on $\Omega$ defined by

$$
\left\langle\Delta_{k}\left(f \omega_{k}\right), \varphi\right\rangle=\left\langle f \omega_{k}, \Delta_{k} \varphi\right\rangle, \quad \varphi \in \mathcal{D}(\Omega) .
$$


Moreover, for any $x \in \mathbb{R}^{d}, N_{k}(x, x)=+\infty$. For more details on the properties of the $\Delta_{k}$-Newton kernel one can see Section 6 in [13].

Let $\Omega$ be a $W$-invariant open subset of $\mathbb{R}^{d}$. Recall that a function $u: \Omega \longrightarrow[-\infty,+\infty[$ is $\Delta_{k}$-subharmonic if (see [13])

1. $u$ is upper semi-continuous on $\Omega$,

2. $u$ is not identically $-\infty$ on each connected component of $\Omega$,

3. $u$ satisfies the volume sub-mean property i.e. for each closed ball $\bar{B}(x, r) \subset \Omega$, we have

$$
u(x) \leq M_{B}^{r}(u)(x):=\frac{1}{m_{k}[B(0, r)]} \int_{\mathbb{R}^{d}} u(y) h_{k}(r, x, y) \omega_{k}(y) d y .
$$

Here $m_{k}$ is the measure $d m_{k}(x):=\omega_{k}(x) d x$ and $y \mapsto h_{k}(r, x, y)$ is the nonnegative compactly supported measurable function given by

$$
h_{k}(r, x, y):=\int_{\mathbb{R}^{d}} \mathbf{1}_{[0, r]}\left(\sqrt{\|x\|^{2}+\|y\|^{2}-2\langle x, z\rangle}\right) d \mu_{y}(z) .
$$

We refer to [11] for more details on the kernel $h_{k}$.

The following result gives some useful facts about the Poisson integral of the $\Delta_{k^{-}}$ Newton kernel:

\section{Proposition 4.1}

i) For each $x \in A$, the function $P_{k, A}\left[N_{k}(x,).\right]$ is the solution of the Dirichlet problem

$$
\begin{cases}\Delta_{k} u=0, & \text { on } A \\ u=N_{k}(x, .) & \text { on } \partial A\end{cases}
$$

ii) The function $(x, y) \mapsto P_{k, A}\left[N_{k}(x,).\right](y)$ is continuous on $A \times \bar{A}$.

iii) For each fixed $y \in A$, the function $x \mapsto P_{k, A}\left[N_{k}(x,).\right](y)$ is $\Delta_{k}$-harmonic in $A$.

Proof: i) If $x \in A$, then the function $N_{k}(x,$.$) is continuous on \partial A$ and by Theorem 3.1, we obtain the first assertion.

ii) At first, we shall prove the following result

Lemma 4.1 The function $(x, y) \mapsto N_{k}(x, y)$ is continuous on $\mathbb{R}^{d} \times \mathbb{R}^{d} \backslash\{(x, g x), \quad x \in$ $\left.\mathbb{R}^{d}, g \in W\right\}$.

Proof: Using the following inequality (see [24] Lemma 4.2)

$$
\forall t>0, \quad p_{k}(t, x, y) \leq \frac{1}{c_{k}(2 t)^{d / 2+\gamma}} \max _{g \in W} e^{-\|x-g y\|^{2} / 4 t},
$$


we can apply the dominated convergence theorem in formula (4.1) to obtain the result of the lemma.

From the first assertion, for each $x \in A, P_{k, A}\left[N_{k}(x,).\right]$ is extendable to a continuous function on $\bar{A}$ with $P_{k, A}\left[N_{k}(x,).\right]=N_{k}(x,$.$) on \partial A$.

Let $\left(x_{0}, y_{0}\right) \in A \times \bar{A}$. For every $(x, y) \in A \times \bar{A}$ we have

$$
\begin{aligned}
\left|P_{k, A}\left[N_{k}(x, .)\right](y)-P_{k, A}\left[N_{k}\left(x_{0}, .\right)\right]\left(y_{0}\right)\right| & \leq\left|P_{k, A}\left[N_{k}(x, .)\right](y)-P_{k, A}\left[N_{k}\left(x_{0}, .\right)\right](y)\right| \\
& +\left|P_{k, A}\left[N_{k}\left(x_{0}, .\right)\right](y)-P_{k, A}\left[N_{k}\left(x_{0}, .\right)\right]\left(y_{0}\right)\right| \\
& \leq \mathbf{P}_{k, 1}\left[\left|K_{x_{0}}(x, .)\right|\right](y)+\mathbf{P}_{k, 2}\left[\left|K_{x_{0}}(x, .)\right|\right](y) \\
& +\left|P_{k, A}\left[N_{k}\left(x_{0}, .\right)\right](y)-P_{k, A}\left[N_{k}\left(x_{0}, .\right)\right]\left(y_{0}\right)\right|
\end{aligned}
$$

where $K_{x_{0}}(x, y):=N_{k}(x, y)-N_{k}\left(x_{0}, y\right)$.

We already know that

$$
\lim _{y \rightarrow y_{0}} P_{k, A}\left[N_{k}\left(x_{0}, .\right)\right](y)=P_{k, A}\left[N_{k}\left(x_{0}, .\right)\right]\left(y_{0}\right) .
$$

Now, let $\varepsilon>0$ and $R>0$ be such that $\bar{B}\left(x_{0}, R\right) \subset A$. Since $(x, \xi) \longmapsto N_{k}(x, \xi)$ is uniformly continuous on $\bar{B}\left(x_{0}, R\right) \times S^{d-1}$, we deduce that there exists $\eta>0$ such that

$$
\forall(x, \xi) \in B\left(x_{0}, \eta\right) \times S^{d-1}, \quad\left|K_{x_{0}}(x, \xi)\right|=\left|N_{k}(x, \xi)-N_{k}\left(x_{0}, \xi\right)\right|<\varepsilon .
$$

Then, using (3.15) as well as the inequalities (3.2) and (3.10), we get for every $x \in B\left(x_{0}, \eta\right)$ and every $y \in A$

$$
\mathbf{P}_{k, 1}\left[\left|K_{x_{0}}(x, .)\right|\right](y) \leq \frac{1}{d_{k}} \int_{S^{d-1}} \mathbf{P}_{k, 1}(y, \xi)\left|K_{x_{0}}(x, \xi)\right| \omega_{k}(\xi) d \sigma(\xi) \leq \varepsilon .
$$

The same idea works if we replace the kernel $\mathbf{P}_{k, 1}$ by $\mathbf{P}_{k, 2}$. Finally, we obtain

$$
\lim _{(x, y) \rightarrow\left(x_{0}, y_{0}\right)} P_{k, A}\left[N_{k}(x, .)\right](y)=P_{k, A}\left[N_{k}\left(x_{0}, .\right)\right]\left(y_{0}\right) .
$$

That is the function $(x, y) \mapsto P_{k, A}\left[N_{k}(x,).\right](y)$ is continuous on $A \times \bar{A}$ as desired.

iii) According to Corollary 4.6 in [12], it is enough to show that the functions $x \mapsto u_{y}(x):=$ $\mathbf{P}_{k, 1}\left[N_{k}(x,).\right](y)$ and $x \mapsto v_{y}(x):=\mathbf{P}_{k, 2}\left[N_{k}(x,).\right](y)$ satisfy the volume-mean property.

Let then $x_{0} \in A$ and $R>0$ such that $\bar{B}\left(x_{0}, R\right) \subset A$. As the kernels $N_{k}, h_{k}$ and $\mathbf{P}_{k, 1}$ are nonnegative, we can use Fubini's theorem to obtain

$$
M_{B}^{R}\left(u_{y}\right)\left(x_{0}\right)=\frac{1}{d_{k}} \int_{S^{d-1}} \mathbf{P}_{k, 1}(y, \xi) M_{B}^{R}\left[N_{k}(., \xi)\right]\left(x_{0}\right) \omega_{k}(\xi) d \sigma(\xi)
$$

But for any $\xi \in S^{d-1}$, the function $N_{k}(., \xi)$ is $\Delta_{k}$-harmonic on $A$. Hence, it satisfies the volume-mean property i.e. $M_{B}^{R}\left[N_{k}(., \xi)\right]\left(x_{0}\right)=N_{k}\left(x_{0}, \xi\right)$. Therefore, we obtain

$$
\begin{aligned}
M_{B}^{R}\left(u_{y}\right)\left(x_{0}\right) & =\frac{1}{d_{k}} \int_{S^{d-1}} \mathbf{P}_{k, 1}(y, \xi) N_{k}\left(x_{0}, \xi\right)\left(x_{0}\right) \omega_{k}(\xi) d \sigma(\xi) \\
& =\mathbf{P}_{k, 1}\left[N_{k}\left(x_{0}, .\right)\right](y)=u_{y}\left(x_{0}\right) .
\end{aligned}
$$


By the same way, we get that $x \mapsto v_{y}(x):=\mathbf{P}_{k, 2}\left[N_{k}(x,).\right](y)$ is also a $\Delta_{k}$-harmonic function in $A$. This proves the desired result.

Definition 4.1 For $x \in A$, the function $G_{k, A}(x,$.$) defined by$

$$
G_{k, A}(x, y):=N_{k}(x, y)-P_{k, A}\left[N_{k}(x, .)\right](y), \quad y \in A,
$$

is called the $\Delta_{k}$-Green function of $A$ with pole $x$.

The $\Delta_{k}$-Green function $G_{k, A}$ has the following properties:

Proposition 4.2 Let $x \in A$. Then

1. The function $G_{k, A}(x,$.$) is \Delta_{k}$-harmonic on $A \backslash W . x$, is $\Delta_{k}$-superharmonic on $A$ and satisfies

$$
-\Delta_{k}\left[G_{k, A}(x, .) \omega_{k}\right]=\delta_{x} \quad \text { in } \quad \mathcal{D}^{\prime}(A) .
$$

2. $G_{k, A}(x, x)=+\infty$ and $G_{k, A}(x, y)<+\infty$ whenever $y \notin W . x$.

3. For every $\xi \in \partial A, \lim _{y \rightarrow \xi} G_{k, A}(x, y)=0$.

4. For every $y \in A, G_{k, A}(x, y)>0$.

5. For every $x, y \in A, G_{k, A}(x, y)=G_{k, A}(y, x)$.

6. For every $x, y \in A$ and $g \in W, G_{k, A}(g x, g y)=G_{k, A}(x, y)$.

7. The zero function is the greatest $\Delta_{k}$-subharmonic minorant of $G_{k, A}(x,$.$) on A$.

8. The function $(x, y) \mapsto G_{k, A}(x, y)$ is continuous on $A \times \bar{A} \backslash\{(x, g x): x \in A, g \in W\}$.

Proof: The first and the second assertions follow from the properties of the $\Delta_{k}$-Newton kernel previously mentioned. In addition, by Proposition 4.1, we easily obtain the third statement.

4) As $\lim _{y \rightarrow \xi \in \partial A} G_{k, A}(x, y)=0$, the weak minimum principle for $\Delta_{k}$-superharmonic functions (see [13], Theorem 3.1) implies that $G_{k, A}(x,) \geq$.0 on $A$.

If $G_{k, A}\left(x, y_{0}\right)=0$ for some $y_{0} \in A$, from the strong maximum principle (see [13]), we must have $G_{k, A}(x,$.$) is the zero function on A$ which is impossible because $G_{k, A}(x, x)=+\infty$. Thus, $G_{k, A}$ is a positive kernel on $A \times A$.

5) Since $N_{k}$ is a symmetric kernel, we have to prove that

$$
\forall x, y \in A, \quad P_{k, A}\left[N_{k}(x, .)\right](y)=P_{k, A}\left[N_{k}(y, .)\right](x) .
$$

Let $y \in A$ and consider the function

$$
H_{y}(x):=P_{k, A}\left[N_{k}(x, .)\right](y)-P_{k, A}\left[N_{k}(y, .)\right](x) .
$$


From Proposition 4.1 , i) and iii), $H_{y}$ is a $\Delta_{k}$-harmonic function in $A$. On the other hand, writing

$$
H_{y}(x)=N_{k}(x, y)-G_{k, A}(x, y)-P_{k, A}\left[N_{k}(y, .)\right](x)
$$

and using the positivity of $G_{k, A}$ as well as the symmetry property of the kernel $N_{k}$, we conclude that

$$
\limsup _{x \mapsto \xi \in \partial A} H_{y}(x) \leq N_{k}(\xi, y)-N_{k}(y, \xi)=0 .
$$

Then, by the weak maximum principle, it yields that $H_{y} \leq 0$ on $A$. That is, we have

$$
\forall x, y \in A, \quad P_{k, A}\left[N_{k}(x, .)\right](y) \leq P_{k, A}\left[N_{k}(y, .)\right](x) .
$$

By interchanging the role of $x$ and $y$, we also get the reverse inequality. Finally, we obtain the desired equality.

6) The result follows immediately from (3.20) and from the relation $N_{k}(g x, g y)=N_{k}(x, y)$, $x, y \in \mathbb{R}^{d}, g \in W$ (see [13]).

7) As $G_{k, A}(x,$.$) is positive on A$, we know that the zero function is a $\Delta_{k}$-subharmonic minorant of $G_{k, A}(x,$.$) .$

Now, let $s$ be a $\Delta_{k}$-subharmonic function on $A$ such that $s \leq G_{k, A}(x,$.$) on A$. Using the statement 3), we obtain $\lim \sup _{z \rightarrow \xi \in \partial A} s(z) \leq 0$. Thus from the weak maximum principle for $\Delta_{k}$-subharmonic functions (see [13]) it yields that $s \leq 0$ on $A$.

8) The result follows immediately from the statement ii) of Proposition 4.1 and Lemma 4.1 .

In the following result, we will express the Green function via the $\Delta_{k}$-spherical harmonics. More precisely, we have

Theorem 4.1 The $\Delta_{k}$-Green function in $A$ is given by

$$
G_{k, A}(x, y)=N_{k}(x, y)-\sum_{n=0}^{+\infty} \frac{a_{k, n}(y)\|x\|^{n}+b_{k, n}(y)\|x\|^{-n-2 \lambda_{k}} \rho^{n}}{d_{k}\left(2 \lambda_{k}+2 n\right)} Z_{k, n}\left(\frac{x}{\|x\|}, y\right) .
$$

We need the following result:

Proposition 4.3 For $x, y \in \mathbb{R}^{d}$ such that $\|y\|<\|x\|$, we have

$$
N_{k}(x, y)=\sum_{n=0}^{+\infty} \frac{\|x\|^{-2 \lambda_{k}}}{d_{k}\left(2 \lambda_{k}+2 n\right)}\|y\|^{n}\|x\|^{-n} Z_{k, n}\left(\frac{x}{\|x\|}, \frac{y}{\|y\|}\right) .
$$

Proof: Let $\|y\|<\|x\|$. From (4.3), we have

$$
\begin{aligned}
N_{k}(x, y) & =\frac{\|x\|^{-2 \lambda_{k}}}{2 d_{k} \lambda_{k}} \int_{\mathbb{R}^{d}}\left(1-\frac{2\langle x, z\rangle}{\|x\|^{2}}+\frac{\|y\|^{2}}{\|x\|^{2}}\right)^{-\lambda_{k}} d \mu_{y}(z) \\
& =\frac{\|x\|^{-2 \lambda_{k}}}{2 d_{k} \lambda_{k}} \int_{\mathbb{R}^{d}} \sum_{n=0}^{+\infty}\|y\|^{n}\|x\|^{-n} \frac{\left(2 \lambda_{k}\right)_{n}}{n !} P_{n}^{\lambda_{k}}\left(\left\langle\frac{x}{\|x\|}, \frac{z}{\|y\|}\right\rangle\right) d \mu_{y}(z) \\
& =\sum_{n=0}^{+\infty} \frac{\|x\|^{-2 \lambda_{k}}}{d_{k}\left(2 \lambda_{k}+2 n\right)}\|y\|^{n}\|x\|^{-n} Z_{k, n}\left(\frac{x}{\|x\|}, \frac{y}{\|y\|}\right) ;
\end{aligned}
$$


where in the second line, we have used the relation (2.3) and the generating relation (see for example [8], p. 18)

$$
\left(1-2 a r+r^{2}\right)^{-\mu}=\sum_{n=0}^{+\infty} \frac{(2 \mu)_{n}}{n !} P_{n}^{\mu}(a) r^{n}, \quad \mu>0, \quad|r|<1,|a| \leq 1
$$

and in the last line, we have used

- the inequality $\sup _{x \in[-1,1]}\left|P_{n}^{\mu}(x)\right| \leq P_{n}^{\mu}(1)$ (see [25], Theorem 7.32.1) and the above generation relation with $a=1$ which allowed as to permute the symbols $\sum$ and $\int$,

- the fact that $\mu_{\frac{y}{\|y\|}}$ is the image measure of $\mu_{y}$ by the dilation $\xi \mapsto \frac{\xi}{\|y\|}$

- the relation $(2.13)$.

Proof of Theorem 4.1: By Theorem 3.1, we have

$$
P_{k, A}\left[N_{k}(x, .)\right](y)=\mathbf{P}_{k, 1}\left[N_{k}(x, .)\right](y)+\mathbf{P}_{k, 2}\left[N_{k}(x, .)\right](y):=I_{1}+I_{2} .
$$

- We have

$$
\begin{aligned}
I_{1} & =\sum_{n=0}^{+\infty} \frac{a_{k, n}(y)}{d_{k}} \int_{S^{d-1}} Z_{k, n}(y, \xi) N_{k}(x, \xi) \omega_{k}(\xi) d \sigma(\xi) \\
& =\sum_{n=0}^{+\infty} \frac{a_{k, n}(y)}{d_{k}} \int_{S^{d-1}} \sum_{m=0}^{+\infty} \frac{\|x\|^{m}}{d_{k}\left(2 \lambda_{k}+2 m\right)} Z_{k, m}\left(\frac{x}{\|x\|}, \xi\right) Z_{k, n}(y, \xi) \omega_{k}(\xi) d \sigma(\xi) \\
& =\sum_{n=0}^{+\infty} a_{k, n}(y) \sum_{m=0}^{+\infty} \frac{\|x\|^{m}}{d_{k}\left(2 \lambda_{k}+2 m\right)} \frac{1}{d_{k}} \int_{S^{d-1}} Z_{k, m}\left(\frac{x}{\|x\|}, \xi\right) Z_{k, n}(y, \xi) \omega_{k}(\xi) d \sigma(\xi) \\
& =\sum_{n=0}^{+\infty} \frac{a_{k, n}(y)\|x\|^{n}}{d_{k}\left(2 \lambda_{k}+2 n\right)} Z_{k, n}\left(\frac{x}{\|x\|}, y\right),
\end{aligned}
$$

where, we have used

-the relation (4.9) with $x \leftarrow y$ and $x \leftarrow \xi \in S^{d-1}$ in the second line;

-the inequalities (3.13) and $\|x\|<1$ in order to the change the symbols $\int$ and $\sum$ in the third line;

-the fact that $\mathcal{H}_{\Delta_{k}, n}\left(\mathbb{R}^{d}\right) \perp \mathcal{H}_{\Delta_{k}, m}\left(\mathbb{R}^{d}\right)$ whenever $m \neq n$ and the reproducing formula (2.10) in the last line.

Note that if $\|x\|>\rho$, then from (4.9) it yields that

$$
\forall \xi \in S^{d-1}, \quad N_{k}(x, \rho \xi)=\sum_{n=0}^{+\infty} \frac{\|x\|^{-2 \lambda_{k}}}{d_{k}\left(2 \lambda_{k}+2 n\right)} \rho^{n}\|x\|^{-n} Z_{k, n}\left(\frac{x}{\|x\|}, \xi\right) .
$$


Consequently, by the same way, we obtain

$$
\begin{aligned}
I_{2} & =\sum_{n=0}^{+\infty} \frac{b_{k, n}(y)}{d_{k}} \int_{S^{d-1}} Z_{k, n}(y, \xi) N_{k}(x, \rho \xi) \omega_{k}(\xi) d \sigma(\xi) \\
& =\sum_{n=0}^{+\infty} \frac{b_{k, n}(y)}{d_{k}} \int_{S^{d-1}} \sum_{m=0}^{+\infty} \frac{\|x\|^{-2 \lambda_{k}-m} \rho^{m}}{d_{k}\left(2 \lambda_{k}+2 m\right)} Z_{k, m}\left(\frac{x}{\|x\|}, \xi\right) Z_{k, n}(y, \xi) \omega_{k}(\xi) d \sigma(\xi) \\
& =\sum_{n=0}^{+\infty} b_{k, n}(y) \sum_{m=0}^{+\infty} \frac{\|x\|^{-2 \lambda_{k}-m} \rho^{m}}{d_{k}\left(2 \lambda_{k}+2 m\right)} \frac{1}{d_{k}} \int_{S^{d-1}} Z_{k, m}\left(\frac{x}{\|x\|}, \xi\right) Z_{k, n}(y, \xi) \omega_{k}(\xi) d \sigma(\xi) \\
& =\sum_{n=0}^{+\infty} \frac{b_{k, n}(y)\|x\|^{-2 \lambda_{k}-n} \rho^{n}}{d_{k}\left(2 \lambda_{k}+2 n\right)} Z_{k, n}\left(\frac{x}{\|x\|}, y\right) .
\end{aligned}
$$

This gives the desired formula (4.8).

Remark 4.1 Using (4.9) and replacing the functions $a_{k, n}$ and $b_{k, n}$ by their expressions, if $x, y \in A$ with $\|y\|<\|x\|$ we can write

$$
G_{k, A}(x, y)=\sum_{n=0}^{+\infty} \frac{\left(\|y\|^{2 \lambda_{k}+2 n}-\rho^{2 \lambda_{k}+2 n}\right)\left(1-\|x\|^{2 \lambda_{k}+2 n}\right)}{d_{k}\left(2 \lambda_{k}+2 n\right)\left(1-\rho^{2 \lambda_{k}+2 n}\right)(\|x\|\|y\|)^{2 \lambda_{k}+n}} Z_{k, n}\left(\frac{x}{\|x\|}, \frac{y}{\|y\|}\right) .
$$

This formula generalizes the classical case (if $k=0,2 \lambda_{0}=d-2$ ) proved in [16].

\section{Applications}

\subsection{Poisson-Jensen formula for $\Delta_{k}$-subharmonic functions}

Our goal now is to prove an analogue of the Poisson-Jensen formula for $\Delta_{k}$-subharmonic functions on $\Omega \supset \bar{A}$. Note that a Poisson-Jensen formula has been proved when $u$ is a $C^{2}-\Delta_{k}$-subharmonic function on $\Omega$ which contains the closed unit ball (see [15]).

Theorem 5.1 Let $u$ be a $\Delta_{k}$-subharmonic function on a $W$-invariant open set $\Omega \supset \bar{A}$. Then,

$$
u(x)=P_{k, A}[u](x)-\int_{A} G_{k, A}(x, y) d \nu_{u}(y), \quad x \in A,
$$

where $\nu_{u}:=\Delta_{k}\left(u \omega_{k}\right)$ is the $\Delta_{k}$-Riesz measure of $u$ (see [13]).

Proof: Let $O$ be a bounded $W$-invariant open set such that $\bar{A} \subset O \subset \bar{O} \subset \Omega$. Using the Riesz decomposition theorem for $\Delta_{k}$-subharmonic functions (see [13]), we deduce that there exists a $\Delta_{k}$-harmonic function $h$ on $O$ such that

$$
\forall x \in O, \quad u(x)=h(x)-\int_{O} N_{k}(x, y) d \nu_{u}(y):=h(x)-s(x) .
$$


Then, we have

$$
\forall x \in A, \quad P_{k, A}[u](x)=P_{k, A}[h](x)-P_{k, A}[s](x) .
$$

From Corollary 3.2, we have $P_{k, A}[h]=h$ on $A$. Moreover, for $x \in A$, we have

$$
P_{k, A}[s](x)=\mathbf{P}_{k, 1}[s](x)+\mathbf{P}_{k, 2}[s](x) .
$$

The crucial part here is to show that

$$
\forall x \notin A, \quad P_{k, A}\left[N_{k}(x, .)\right]=N_{k}(x, .) \quad \text { on } A .
$$

Assume this relation for the moment. By Fubini's theorem, we have

$$
\begin{aligned}
\mathbf{P}_{k, 1}[s](x) & :=\frac{1}{d_{k}} \int_{S^{d-1}} \mathbf{P}_{k, 1}(x, \xi) s(\xi) \omega_{k}(\xi) d \sigma(\xi) \\
& =\frac{1}{d_{k}} \int_{O} \int_{S^{d-1}} \mathbf{P}_{k, 1}(x, \xi) N_{k}(\xi, y) \omega_{k}(\xi) d \sigma(\xi) d \nu_{u}(y) \\
& =\int_{O} \mathbf{P}_{k, 1}\left[N_{k}(y, .)\right](x) d \nu_{u}(y) .
\end{aligned}
$$

By the same way, we also have

$$
\mathbf{P}_{k, 2}[s](x)=\int_{O} \mathbf{P}_{k, 2}\left[N_{k}(y, .)\right](x) d \nu_{u}(y)
$$

The above relations as well as (5.2) imply that

$$
\begin{aligned}
P_{k, A}[s](x) & =\int_{O} P_{k, A}\left[N_{k}(y, .)\right](x) d \nu_{u}(y) \\
& =\int_{A} P_{k, A}\left[N_{k}(y, .)\right](x) d \nu_{u}(y)+\int_{O \backslash A} P_{k, A}\left[N_{k}(y, .)\right](x) d \nu_{u}(y) \\
& =\int_{A}\left(N_{k}(x, y)-G_{k, A}(x, y)\right) d \nu_{u}(y)+\int_{O \backslash A} N_{k}(x, y) d \nu_{u}(y) \\
& =\int_{O} N_{k}(x, y) d \nu_{u}(y)-\int_{A} G_{k, A}(x, y) d \nu_{u}(y)
\end{aligned}
$$

This implies the desired Poisson-Jensen formula. Now, it remains to prove (5.2). We will distinguish three cases.

First case: $x \notin \bar{A}$. As $N_{k}(x,$.$) is \Delta_{k}$-harmonic on $A$ and continuous on $\bar{A}$, we deduce by Corollary 3.2 that $P_{k, A}\left[N_{k}(x,).\right]=N_{k}(x,$.$) on A$.

Second case: $x \in S^{d-1}$. For $\varepsilon>0$ small enough, the function $N_{k}((1+\varepsilon) x,$.$) is \Delta_{k^{-}}$ harmonic in the open ball $B(0,1+\varepsilon) \supset \bar{A}$. Therefore, again by Corollary 3.2, we obtain

$$
\forall y \in A, \quad N_{k}((1+\varepsilon) x, y)=P_{k, A}\left[N_{k}((1+\varepsilon) x, .)\right](y) .
$$

Clearly we have $\lim _{\varepsilon \rightarrow 0} N_{k}((1+\varepsilon) x, y)=N_{k}(x, y)$ for every fixed $y \in A$. Moreover, using (4.3) and the fact that supp $\mu_{y} \subset \bar{B}(0,\|y\|)$ we can see that

$$
N_{k}((1+\varepsilon) x, y) \leq N_{k}(x, y), \quad \text { whenever } \quad\|y\| \leq\|x\| .
$$


Consequently, we can use the dominated convergence theorem to obtain

$$
\forall y \in A, \quad \lim _{\varepsilon \rightarrow 0} P_{k, A}\left[N_{k}((1+\varepsilon) x, .)\right](y)=P_{k, A}\left[N_{k}(x, .)\right](y) .
$$

Hence, letting $\varepsilon \longrightarrow 0$ in the relation (5.3), we get the result in this case.

Third case: $x \in S(0, \rho)$. Let $0<\varepsilon<1 / 2$. In this case, the function $N_{k}((1-\varepsilon) x,$.$) is$ $\Delta_{k}$-harmonic in $\mathbb{R}^{d} \backslash \bar{B}(0,(1-\varepsilon) \rho) \supset \bar{A}$ and then from Corollary 3.2 we deduce that

$$
\forall y \in A, \quad N_{k}((1-\varepsilon) x, y)=P_{k, A}\left[N_{k}((1-\varepsilon) x, .)\right](y) .
$$

Note that from (2.3), we can write

$$
N_{k}(x, y)=\frac{1}{2 d_{k} \lambda_{k}} \int_{\mathbb{R}^{d}}\left(\sum_{g \in W} \lambda_{g}(z)\|x-g y\|^{2}\right)^{-\lambda_{k}} d \mu_{y}(z),
$$

where for every $z \in \operatorname{supp} \mu_{y}$, the nonnegative numbers $\lambda_{g}(z)$ are such that $\sum_{g \in W} \lambda_{g}(z)=$ 1. Using the above relation we easily see that

$$
N_{k}((1-\varepsilon) x, y) \leq 2^{2 \lambda_{k}} N_{k}(x, y), \quad \text { whenever } \quad\|x\| \leq\|y\|,
$$

Finally by same way as in the second case we obtain the result.

\subsection{Positive solution of $\Delta_{k}$-nonlinear elliptic problem on the annulus}

In this section, we will investigate the positive continuous solutions of the semilinear problem

$$
\Delta_{k}\left(u \omega_{k}\right)=\phi(., u) \omega_{k} \quad \text { in } \quad \mathcal{D}^{\prime}(A),
$$

in the sense that

$$
\forall \varphi \in \mathcal{D}(A), \quad \int_{A} u(x) \Delta_{k} \varphi(x) \omega_{k}(x) d x=\int_{A} \varphi(x) \phi(x, u(x)) \omega_{k}(x) d x .
$$

We will suppose that $\phi$ has the form $\phi=\phi_{1} \phi_{2}: A \times[0,+\infty[\longrightarrow[0,+\infty[$ with

- $\phi_{1}$ is a nonnegative bounded measurable function on $A$.

- $\phi_{2}$ is a nonnegative and nondecreasing continuous function on $\left[0,+\infty\left[\right.\right.$ with $\phi_{2}(0)=0$.

In [3], by using some tools from probabilistic potential theory, the authors have studied the positive solution on the unit ball $B$ of the semilinear problem

$$
\Delta_{k}(u)=\varphi(u) \quad \text { in } \mathcal{D}^{\prime}(B) \text { and } \quad u=f \quad \text { on } \partial B .
$$

Let us denote by $\mathcal{C}^{+}(\bar{A})$ the convex cone of nonnegative and continuous functions on $A$.

Theorem 5.2 Let $\phi=\phi_{1} \phi_{2}$ as above. Then, for every $f \in \mathcal{C}^{+}(\partial A)$, the semilinear Dirichlet problem

$$
\begin{cases}\Delta_{k}\left(u \omega_{k}\right)=\phi(., u) \omega_{k}, & \text { in } \mathcal{D}^{\prime}(A) \\ u=f, & \text { on } \partial A\end{cases}
$$


admits one and only one solution $u \in \mathcal{C}^{+}(\bar{A})$. Furthermore, we have

$$
\forall x \in A, \quad u(x)+\int_{A} G_{k, A}(x, y) \phi(y, u(y)) \omega_{k}(y) d y=P_{k, A}[f](x) .
$$

We begun by showing the uniqueness of the solution. This fact follows immediately from the following maximum principle type result:

Lemma 5.1 Let $u, v \in \mathcal{C}(A)$ and let $\phi$ be a function satisfying the above conditions. If

$$
\left\{\begin{array}{l}
\Delta_{k}\left(u \omega_{k}\right)-\phi(., u) \omega_{k} \leq \Delta_{k}\left(v \omega_{k}\right)-\phi(., v) \omega_{k}, \quad \text { in } \mathcal{D}^{\prime}(A), \\
\limsup _{x \rightarrow y \in \partial A}(v-u)(x) \leq 0
\end{array}\right.
$$

then $v \leq u$ in $A$.

Proof: Let $U$ be the upper semi-continuous function defined by

$$
U(x)= \begin{cases}v(x)-u(x), & \text { if } x \in A \\ \limsup _{y \rightarrow x \in \partial A}(v-u)(y), & \text { if } x \in \partial A\end{cases}
$$

and $x_{0} \in \bar{A}$ be such that $U\left(x_{0}\right)=\max _{\bar{A}} U$.

We have to prove that $U\left(x_{0}\right) \leq 0$. We suppose the contrary i.e. $U\left(x_{0}\right)>0$. As $U \leq 0$ on $\partial A$, this implies that $x_{0} \notin \partial A$.

Let $O$ be the nonempty open set given by

$$
O:=\{x \in A: \quad U(x)>0\}
$$

and $\Omega$ be the connected component of $x_{0}$ in $O$ which is also an open set of $\mathbb{R}^{d}$.

To get a contradiction, we claim that it is suffices to establish that

$$
U=U\left(x_{0}\right) \quad \text { on } \quad \Omega .
$$

Indeed,

- If $\Omega=O$ (i.e. $O$ is connected), then (5.5) holds on $O=\Omega$. But

$$
\partial O \subset \partial A \cup(A \backslash O)=\{x \in \bar{A}, U(x) \leq 0\} .
$$

Consequently, using the fact that $U$ is upper semi-continuous and (5.5), we get

$$
\forall x \in \partial O, \quad U(x)=\limsup _{y \rightarrow x, y \in O} U(y)=U\left(x_{0}\right) .
$$

Thus, we obtain a contradiction.

- If $\Omega \neq O$, then as $\Omega$ is a connected component of $O$ we have $\partial \Omega \cap O=\emptyset$. Therefore, we have $\partial \Omega \subset \partial A \cup A \backslash O$ and as above we get a contradiction. 
Now, our aim is to prove that $U=U\left(x_{0}\right)$ on $\Omega$. For this, we introduce the nonempty closed set

$$
\Omega_{0}:=\left\{x \in \Omega: \quad U(x)=U\left(x_{0}\right)\right\} .
$$

Note that

$$
\Omega=\left(\Omega \cap \cup_{\alpha \in \mathcal{R}} H_{\alpha}\right) \cup\left(\Omega \backslash \cup_{\alpha \in \mathcal{R}} H_{\alpha}\right)=\left(\Omega \cap \cup_{\alpha \in \mathcal{R}} H_{\alpha}\right) \cup\left(\cup_{g \in W} \Omega \cap g . \mathbf{C}\right),
$$

where $\mathbf{C}$ is a fixed Weyl chamber and $g \mathbf{C}, g \in W$, are the connected components of $\mathbb{R}^{d} \backslash \cup_{\alpha \in \mathcal{R}} H_{\alpha}$.

Fix $\xi \in \Omega_{0}$ and $R>0$ such that the open ball $B(\xi, R)$ is contained in $\Omega$. We will distinguish three possible locations of $\xi$ depending on the sets $E_{1}:=\left\{\alpha \in \mathcal{R}, \quad U\left(\sigma_{\alpha} \xi\right)=U(\xi)\right\}$ and $E_{2}:=\left\{\alpha \in \mathcal{R}, \quad \xi \in H_{\alpha}\right\} \subset E_{1}$.

First case: $E_{1}=\mathcal{R}$. This implies that $U(g \xi)=U(\xi)>0$ for all $g \in W$. Moreover, clearly there exists $r \in] 0, R]$ such that

$$
\forall g \in W, \forall x \in B(g \xi, r), \quad U(x) \geq 0 .
$$

Consider the $W$-invariant continuous function $U^{W}$ defined on $A$ by

$$
U^{W}(x):=\frac{1}{|W|} \sum_{g \in W} g \cdot U(x)=\frac{1}{|W|} \sum_{g \in W} U\left(g^{-1} x\right) .
$$

We easily see that $U^{W}$ has a maximum at the point $\xi$ with $U^{W}(\xi)=U(\xi)=U\left(x_{0}\right)$. Furthermore, using the $W$-invariance property of $\Delta_{k}$ (i.e. $g \circ \Delta_{k}=\Delta_{k} \circ g$ ) and the hypothesis of the lemma, we obtain

$$
\Delta_{k}\left(U^{W} \omega_{k}\right)=\frac{1}{|W|} \sum_{g \in W} g \cdot\left[\Delta_{k}\left(U \omega_{k}\right)\right] \geq \frac{1}{|W|} \sum_{g \in W} g \cdot\left[(\phi(., v)-\phi(., u)) \omega_{k}\right] \quad \text { in } \quad \mathcal{D}^{\prime}(A) .
$$

Now, since $U \geq 0$ on $B^{W}(\xi, r)=\cup_{g \in W} B(g \xi, r)$ (from (5.6)) and $\phi_{2}$ is nondecreasing, we deduce that

$$
\Delta_{k}\left(U^{W} \omega_{k}\right) \geq 0 \quad \text { in } \quad \mathcal{D}^{\prime}\left(B^{W}(\xi, r)\right) .
$$

That is $U$ is weakly $\Delta_{k}$-subharmonic on $B^{W}(\xi, r)$. But the continuity of $U$ and the Weyl lemma for $\Delta_{k}$-subharmonic functions (see [13], Theorem 5.2) imply that $U^{W}$ is strongly $\Delta_{k}$-subharmonic on the open $W$-invariant set $B^{W}(\xi, r)$.

Now, if we follow the proof of the strong maximum principle in [13] for the $W$-invariant $\Delta_{k}$-subharmonic function $U^{W}$, then we conclude that

$$
U=U(\xi)=U\left(x_{0}\right), \quad \text { on } \quad B(\xi, r) .
$$

Hence, we have $B(\xi, r) \subset \Omega_{0}$.

Second case: $E_{1} \neq \mathcal{R}$ and $E_{2}=\emptyset$ i.e. $\xi \notin \cup_{\alpha \in \mathcal{R}} H_{\alpha}$. So there is a unique $g_{0} \in W$ such that $\xi \in \Omega \cap g_{0} \mathbf{C}$. Clearly, we can suppose that $B(\xi, R) \subset \Omega \cap g_{0} \mathbf{C}$. 
Let $U^{W}$ be the W-invariant continuous function defined on $B^{W}(\xi, R):=\cup_{g \in W} B(g \xi, R)$ by

$$
U^{W}(x)=g \cdot U(x):=U\left(g^{-1} . x\right) \quad \text { whenever } \quad x \in B(g \xi, R) .
$$

We are going to establish that the function $U^{W}$ is $\Delta_{k}$-subharmonic on $B^{W}(\xi, r)$ for some $r>0$ will be chosen later. Again from the continuity of $U^{W}$ and the Weyl lemma, it is enough to show that $U^{W}$ is $\Delta_{k}$-subharmonic in distributional sense.

- Firstly, we have the following decomposition

$$
U^{W}=\sum_{i=1}^{n} U^{W} \mathbf{1}_{B\left(g_{i} \xi, R\right)}=\sum_{i=1}^{n} g_{i} \cdot\left[U \mathbf{1}_{B(\xi, R)}\right],
$$

where $g_{1}=i d, g_{2}, \ldots, g_{n} \in W$ are such that $\mathbf{1}_{B^{W}(\xi, R)}=\sum_{i=1}^{n} \mathbf{1}_{B\left(g_{i} \xi, R\right)}$.

- Secondly, for $f \in \mathcal{C}^{2}\left(B^{W}(\xi, R)\right)$, we can write $\Delta_{k}=L_{k}-A_{k}$ where

$$
L_{k} f(x)=\Delta f(x)+2 \sum_{\alpha \in \mathcal{R}_{+}} k(\alpha) \frac{\langle\nabla f(x), \alpha\rangle}{\langle\alpha, x\rangle}
$$

and

$$
A_{k} f(x)=\sum_{\alpha \in \mathcal{R}_{+}} k(\alpha)\|\alpha\|^{2} \frac{f(x)-f\left(\sigma_{\alpha}(x)\right)}{\langle\alpha, x\rangle^{2}} .
$$

- For $\varphi \in \mathcal{D}\left(B^{W}(\xi, R)\right)$ nonnegative, we have

$$
\begin{aligned}
\left\langle\Delta_{k}\left(U^{W} \omega_{k}\right), \varphi\right\rangle & =\sum_{i=1}^{n}\left\langle\Delta_{k}\left(g_{i} \cdot\left[U \mathbf{1}_{B(\xi, R)}\right] \omega_{k}\right), \varphi\right\rangle=\sum_{i=1}^{n}\left\langle g_{i} \cdot\left[U \omega_{k} \mathbf{1}_{B(\xi, R)}\right], \Delta_{k} \varphi\right\rangle \\
& =\sum_{i=1}^{n}\left\langle g_{i} \cdot\left[U \omega_{k} \mathbf{1}_{B(\xi, R)}\right], L_{k} \varphi-A_{k} \varphi\right\rangle \\
& =\sum_{i=1}^{n}\left\langle U \omega_{k},\left(L_{k}\left[g_{i}^{-1} \cdot \varphi\right]\right) \mathbf{1}_{B(\xi, R)}\right\rangle-\left\langle\sum_{i=1}^{n} g_{i} \cdot\left[U \mathbf{1}_{B(\xi, R)}\right] \omega_{k}, A_{k}(\varphi)\right\rangle \\
& =\sum_{i=1}^{n}\left\langle U \omega_{k}, L_{k}\left(\left[g_{i}^{-1} \cdot \varphi\right] \mathbf{1}_{B(\xi, R)}\right)\right\rangle-\underbrace{\left\langle U^{W} \omega_{k}, A_{k}(\varphi)\right\rangle}_{=0} \\
& =\sum_{i=1}^{n}\left\langle U \omega_{k}, \Delta_{k}\left(\left[g_{i}^{-1} \cdot \varphi\right] \mathbf{1}_{B(\xi, R)}\right)+A_{k}\left(\left[g_{i}^{-1} \cdot \varphi\right] \mathbf{1}_{B(\xi, R)}\right)\right\rangle \\
& =\sum_{i=1}^{n}\left\langle\Delta_{k}\left(U \omega_{k}\right),\left[g_{i}^{-1} \cdot \varphi\right] \mathbf{1}_{B(\xi, R)}\right\rangle+\sum_{i=1}^{n}\left\langle U \omega_{k}, A_{k}\left(\left[g_{i}^{-1} \cdot \varphi\right] \mathbf{1}_{B(\xi, R)}\right)\right\rangle \\
& \geq \sum_{i=1}^{n}\left\langle[\phi(., v)-\phi(., u)] \omega_{k},\left[g_{i}^{-1} \cdot \varphi\right] \mathbf{1}_{B(\xi, R)}\right\rangle+\sum_{i=1}^{n}\left\langle U \omega_{k}, A_{k}\left(\left[g_{i}^{-1} \cdot \varphi\right] \mathbf{1}_{B(\xi, R)}\right)\right\rangle \\
& \geq \sum_{i=1}^{n}\left\langle U \omega_{k}, A_{k}\left(\left[g_{i}^{-1} \cdot \varphi\right] \mathbf{1}_{B(\xi, R)}\right)\right\rangle,
\end{aligned}
$$


where we have used

- the fact that $L_{k}$ commutes with the $W$-action i.e. $L_{k} \circ g=g \circ L_{k}, g \in W$, in the third line and the fact that it preserves the support in the forth line,

- the $W$-invariance property of $U^{W}$ which implies that $\left\langle U^{W} \omega_{k}, A_{k}(\varphi)\right\rangle=0$ in the forth line,

- the decomposition $L_{k}=\Delta_{k}+A_{k}$ in the fifth line,

- the fact that $\left[g_{i}^{-1} \cdot \varphi\right] \mathbf{1}_{B(\xi, R)} \in \mathcal{D}(B(\xi, R))$ in the sixth line,

- the hypothesis of the lemma in the seventh line,

- the nondecreasing property of $\phi_{2}$ in the last line.

- As $A_{k}$ is a symmetric operator in the sense that $\left\langle A_{k}(f) \omega_{k}, \psi\right\rangle=\left\langle f \omega_{k}, A_{k}(\psi)\right\rangle$, it yields that

$$
\left\langle\Delta_{k}\left(U^{W} \omega_{k}\right), \varphi\right\rangle \geq \sum_{i=1}^{n}\left\langle A_{k}(U) \omega_{k},\left[g_{i}^{-1} \cdot \varphi\right] \mathbf{1}_{B(\xi, R)}\right\rangle,
$$

Clearly $A_{k}(U)(\xi) \geq 0$. But, since $E_{1} \neq \mathcal{R}$, we must have $A_{k}(U)(\xi) \geq 0$. Hence, there exists $r>0$ such that $A_{k}(U)>0$ on $B(\xi, r)$.

Thus, $\Delta_{k}\left(U^{W} \omega_{k}\right) \geq 0$ in $\mathcal{D}^{\prime}\left(B^{W}(\xi, r)\right)$ i.e. $U^{W}$ is weakly $\Delta_{k}$-subharmonic on $B^{W}(\xi, r)$ as desired.

Now, again, if we follow the proof of the strong maximum principle in [13] for the $W$ invariant $\Delta_{k}$-subharmonic function $U^{W}$, then we conclude that

$$
U=U(\xi)=U\left(x_{0}\right) \quad \text { on } \quad B(\xi, r) .
$$

That is $B(\xi, r) \subset \Omega_{0}$.

Third case: $E_{1} \neq \mathcal{R}$ and $E_{2} \neq \emptyset$. Let $W^{\prime} \subsetneq W$ be the isotropy group of $\xi$. Here, we choose $R>0$ under the further following assumption

$$
3 R \leq \min _{\widetilde{g} \in W / W^{\prime}, g \neq i d}\|\xi-g \xi\|, \quad \text { with } \quad W / W^{\prime}:=\left\{\widetilde{g}=g W^{\prime}, g \in W\right\} .
$$

Let $S$ be the $W^{\prime}$-invariant continuous function defined on $A$ by $S=\frac{1}{\left|W^{\prime}\right|} \sum_{g^{\prime} \in W^{\prime}} g^{\prime} . U$.

- Clearly, $S$ has a maximum at the point $\xi$ with $S(\xi)=U(\xi)=U\left(x_{0}\right)$. Furthermore, using the $W$-invariance property of $\Delta_{k}$ as well as the hypothesis the lemma, we get

$$
\Delta_{k}\left(S \omega_{k}\right) \geq \frac{1}{\left|W^{\prime}\right|} \sum_{g^{\prime} \in W^{\prime}} g^{\prime} \cdot(\phi(., v)-\phi(., u)) \omega_{k} \quad \text { in } \quad \mathcal{D}^{\prime}(A) .
$$

- Now, consider the $W$-invariant continuous function $S^{W}$ defined on

$$
B^{W}(\xi, R):=\cup_{g \in W} B(g \xi, R)=\cup_{\widetilde{g} \in W / W^{\prime}} B(g \xi, R)
$$

by

$$
S^{W}(x):=g \cdot S(x)=S\left(g^{-1} \cdot x\right) \quad \text { whenever } \quad x \in B(g \xi, R) \quad \text { and } \widetilde{g} \in W / W^{\prime} .
$$

Note that thanks to the previous condition on $R$, the function $S^{W}$ is well defined.

- Let $\widetilde{g_{1}}=\widetilde{i d}$ and $\widetilde{g_{2}}, \ldots, \widetilde{g_{m}} \in W / W^{\prime}$ such that $\mathbf{1}_{B^{W}(\xi, R)}=\sum_{i=1}^{m} \mathbf{1}_{B\left(g_{i} \xi, R\right)}$ and then we can write

$$
S^{W}=\sum_{i=1}^{m} S^{W} \mathbf{1}_{B\left(g_{i} \xi, R\right)}=\sum_{i=1}^{m} g_{i} \cdot\left[S \mathbf{1}_{B(\xi, R)}\right] .
$$


- Let $\varphi \in \mathcal{D}\left(B^{W}(\xi, R)\right)$ be nonnegative. Following the same idea as in the second case (where we replace $U$ by $S$ and $U^{W}$ by $S^{W}$ ) and using (5.7) we see that we can obtain

$$
\left\langle\Delta_{k}\left(S^{W} \omega_{k}\right), \varphi\right\rangle \geq \sum_{i=1}^{m}\left\langle S \omega_{k}, A_{k}\left(\psi_{i}\right)\right\rangle, \quad \text { with } \quad \psi_{i}=\left[g_{i}^{-1} \cdot \varphi\right] \mathbf{1}_{B(\xi, R)} .
$$

On the other hand, the $W^{\prime}$-invariance property of the function $S$ implies that

$$
\forall i=1, \ldots, m, \forall \alpha \in E_{2}, \quad \int_{B^{W}(\xi, R)} S(x) \frac{\psi_{i}(x)-\psi_{i}\left(\sigma_{\alpha} x\right)}{\langle\alpha, x\rangle^{2}} \omega_{k}(x) d x=0 .
$$

Hence, for every $i=1, \ldots, m$ we have

$$
\begin{aligned}
\left\langle S \omega_{k}, A_{k}\left(\psi_{i}\right)\right\rangle & =\sum_{\alpha \in \mathcal{R}_{+} \backslash E_{2}} k(\alpha)\|\alpha\|^{2} \int_{B^{W}(\xi, R)} S(x) \frac{\psi_{i}(x)-\psi_{i}\left(\sigma_{\alpha} x\right)}{\langle\alpha, x\rangle^{2}} \omega_{k}(x) d x \\
& =\sum_{\alpha \in \mathcal{R}_{+} \backslash E_{2}} k(\alpha)\|\alpha\|^{2} \int_{B(\xi, R)} \frac{S(x)-S\left(\sigma_{\alpha} x\right)}{\langle\alpha, x\rangle^{2}}\left[g_{i}^{-1} \cdot \varphi\right](x) \omega_{k}(x) d x \\
& =\left\langle A_{k}(S) \omega_{k},\left[g_{i}^{-1} \cdot \varphi\right] \mathbf{1}_{B(\xi, R)}\right\rangle
\end{aligned}
$$

As $E_{1} \neq \mathcal{R}$ and

$$
S(\xi)-S\left(\sigma_{\alpha} \xi\right)=\frac{1}{\left|W^{\prime}\right|} \sum_{g^{\prime} \in W^{\prime}}\left(U(\xi)-U\left(\sigma_{g^{\prime-1} . \alpha} \xi\right)\right) \geq 0
$$

we deduce that $A_{k}(S)(\xi)>0$. Consequently, there exists $r>0$ such that $A_{k}(S) \geq 0$ on $B(\xi, r)$. This fact, (5.8), the continuity of $S$ and the Weyl lemma show that $S^{W}$ is $\Delta_{k}$-subharmonic on $B^{W}(\xi, r)$.

Now, by the strong maximum principle, we obtain

$$
S=S(\xi)=U\left(x_{0}\right) \quad \text { on } \quad B(\xi, r) .
$$

Thus, we get

$$
U=U(\xi)=U\left(x_{0}\right) \quad \text { on } \quad B(\xi, r) .
$$

This completes the proof of the lemma.

The main tool to establish the existence of a solution of the boundary problem (5.4) is the Schauder fixed point theorem. In order to apply this theorem, we will prove the following intermediate result:

Proposition 5.1 Let $f$ be a bounded function on $A$ and $G_{k, A}[f]$ be $\Delta_{k}$-Green potential of $f$ on $A$ given by

$$
G_{k, A}[f](x):=\int_{A} G_{k, A}(x, y) f(y) \omega_{k}(y) d y, \quad x \in A .
$$

Then $G_{k, A}[f]$ belongs to $\mathcal{C}_{0}(A)$. Moreover, we have

$$
-\Delta_{k}\left(G_{k, A}[f] \omega_{k}\right)=f \omega_{k} \quad \text { in } \quad \mathcal{D}^{\prime}(A) .
$$


Before proving this result, we need to show the following lemma:

Lemma 5.2 We have

$$
\lim _{r \rightarrow 0} \sup _{x \in A} \eta_{x, r}=0, \quad \text { with } \quad \eta_{x, r}:=\int_{B^{W}(x, r)} N_{k}(x, y) \omega_{k}(y) d y
$$

and $B^{W}(x, r):=\cup_{g \in W} B(g x, r)$.

Proof: Let $x \in A=A_{\rho, 1}$ and $\left.r \in\right] 0, \rho\left[\right.$. Since $N_{k}(x,$.$) is \Delta_{k}$-harmonic on $\mathbb{R}^{d} \backslash W . x$ and $\Delta_{k}$-superharmonic in $\mathbb{R}^{d}$, by the (super-) mean volume property (4.4) we deduce that

$$
\begin{aligned}
0 \leq \eta_{x, r} & \leq \int_{B(0, r+\|x\|) \backslash B(0,\|x\|-r)} N_{k}(x, y) \omega_{k}(y) d y \\
& =\int_{B(0, r+\|x\|)} N_{k}(x, y) \omega_{k}(y) d y-\int_{B(0,\|x\|-r)} N_{k}(x, y) \omega_{k}(y) d y \\
& \leq m_{k}[B(0, r+\|x\|)] N_{k}(x, 0)-m_{k}[B(0,\|x\|-r)] N_{k}(x, 0) \\
& =C N_{k}(x, 0)\left[(\|x\|+r)^{d+2 \gamma}-(\|x\|-r)^{d+2 \gamma}\right] \\
& \leq C \frac{\rho^{-2 \lambda_{k}}}{2 d_{k} \lambda_{k}}\left[(\|x\|+r)^{d+2 \gamma}-(\|x\|-r)^{d+2 \gamma}\right] .
\end{aligned}
$$

This shows that $\lim _{r \rightarrow 0} \sup _{x \in A} \eta_{x, r}=0$ as desired.

Proof of Proposition 5.1: We can suppose that $f$ is nonnegative. Let $\varepsilon>0$. From (5.11), there exists $r>0$ such that

$$
\forall x \in A, \quad \eta_{x, 2 r}<\varepsilon .
$$

- First, we will prove that $G_{k, A}[f](x) \longrightarrow 0$ when $x$ tends to $\partial A$. Let $x \in A$. By (5.12) we have

$$
\begin{aligned}
G_{k, A}[f](x) & =\int_{A} G_{k, A}(x, y) f(y) \omega_{k}(y) d y \\
& =\int_{A \cap B^{W}(x, r)} G_{k, A}(x, y) f(y) \omega_{k}(y) d y+\int_{A \backslash B^{W}(x, r)} G_{k, A}(x, y) f(y) \omega_{k}(y) d y \\
& \leq\|f\|_{\infty} \eta_{x, r}+\|f\|_{\infty} \int_{A \backslash B^{W}(x, r)} G_{k, A}(x, y) \omega_{k}(y) d y \\
& \leq \varepsilon\|f\|_{\infty}+\|f\|_{\infty} \int_{A \backslash B^{W}(x, r)} G_{k, A}(x, y) \omega_{k}(y) d y
\end{aligned}
$$

Since for every $z \in \operatorname{supp} \mu_{y} \subset C o(y)$, we can write

$$
\|x\|^{2}+\|y\|^{2}-2\langle x, z\rangle=\sum_{g \in W} \lambda_{g}(z)\|x-g y\|^{2},
$$

with $\lambda_{g}(z) \geq 0$ and $\sum_{g \in W} \lambda_{g}(z)=1$, we deduce that

$$
\forall y \in A \backslash B^{W}(x, r), \quad 0 \leq G_{k, A}(x, y) \leq N_{k}(x, y) \leq \frac{r^{-2 \lambda_{k}}}{2 d_{k} \lambda_{k}} .
$$


Hence, we can apply the dominated convergence theorem to obtain

$$
\lim _{x \rightarrow \xi \in \partial A} \int_{A \backslash B^{W}(x, r)} G_{k, A}(x, y) \omega_{k}(y) d y=0 .
$$

- Now, we will prove that $G_{k, A}[f]$ is continuous on $A$. Fix $x_{0} \in A$ and assume that $\bar{B}\left(x_{0}, 2 r\right) \subset A$. Since $f$ is bounded, it is enough to prove that

$$
\lim _{x \rightarrow x_{0}} \int_{A}\left|G_{k, A}(x, y)-G_{k, A}\left(x_{0}, y\right)\right| \omega_{k}(y) d y=0
$$

For any $x \in B\left(x_{0}, r\right)$, we have

$$
\begin{aligned}
\int_{A}\left|G_{k, A}(x, y)-G_{k, A}\left(x_{0}, y\right)\right| \omega_{k}(y) d y & \leq \int_{B^{W}\left(x_{0}, r\right)}\left|G_{k, A}(x, y)-G_{k, A}\left(x_{0}, y\right)\right| \omega_{k}(y) d y \\
& +\int_{A \backslash B^{W}\left(x_{0}, r\right)}\left|G_{k, A}(x, y)-G_{k, A}\left(x_{0}, y\right)\right| \omega_{k}(y) d y \\
& =I_{1}\left(x, x_{0}\right)+I_{2}\left(x, x_{0}\right) .
\end{aligned}
$$

As $B^{W}\left(x_{0}, r\right) \subset B^{W}(x, 2 r)$, by (5.12) we have

$$
\begin{aligned}
I_{1}\left(x, x_{0}\right) & \leq \int_{B^{W}\left(x_{0}, r\right)} N_{k}(x, y) \omega_{k}(y) d y+\int_{B^{W}\left(x_{0}, r\right)} N_{k}\left(x_{0}, y\right) \omega_{k}(y) d y \\
& \leq \eta_{x, 2 r}+\eta_{x_{0}, r} \leq 2 \varepsilon .
\end{aligned}
$$

In addition, by the property 8) of Proposition 4.2 we know that the function $(x, y) \longmapsto$ $G_{k, A}(x, y)$ is continuous on the compact set $\bar{B}^{W}\left(x_{0}, r\right) \times\left(\bar{A} \backslash B^{W}\left(x_{0}, r\right)\right)$. Thus, there exists $\theta>0$ such that for every $x \in B\left(x_{0}, \theta\right)$ and every $y \in A \backslash B^{W}\left(x_{0}, r\right)$, we have

$$
\left|G_{k, A}(x, y)-G_{k, A}\left(x_{0}, y\right)\right| \leq \varepsilon .
$$

This implies that

$$
\forall x \in B\left(x_{0}, \theta\right), \quad I_{2}\left(x, x_{0}\right) \leq \varepsilon \int_{A} \omega_{k}(y) d y .
$$

Finally, we conclude that $G_{k, A}[f] \in \mathcal{C}_{0}(A)$.

- Let $\varphi \in \mathcal{D}(A)$. Using Fubini's theorem, the symmetry property of the Green function and (4.7) we get

$$
\begin{aligned}
-\left\langle\Delta_{k}\left(G_{k, A}[f] \omega_{k}\right), \varphi\right\rangle & =-\int_{A} f(y)\left\langle\Delta_{k}\left(G_{k, A}(., y) \omega_{k}\right), \varphi\right\rangle \omega_{k}(y) d y \\
& =\int_{A} f(y) \varphi(y) \omega_{k}(y) d y .
\end{aligned}
$$

This completes the proof. 
Proof of Theorem 5.2: Fix $f \in \mathcal{C}^{+}(\partial A)$ and

$$
\begin{aligned}
c_{1} & :=\inf _{x \in \bar{A}}\left(P_{k, A}[f](x)-G_{k, A}\left[\phi\left(., c_{2}\right)\right](x)\right) \\
& =\inf _{x \in \bar{A}}\left(P_{k, A}[f](x)-\phi_{2}\left(c_{2}\right) G_{k, A}\left[\phi_{1}\right](x)\right), \quad \text { with } \quad c_{2}:=\max _{\bar{A}} P_{k, A}[f] .
\end{aligned}
$$

Let us consider the bounded, closed and convex set

$$
\mathcal{M}:=\left\{u \in \mathcal{C}(\bar{A}): \quad c_{1} \leq u \leq c_{2}\right\}
$$

endowed the uniform topology and the map $T: \mathcal{C}(\bar{A}) \longrightarrow \mathcal{C}(\bar{A})$ defined by

$$
T(u):=P_{k, A}[f]-G_{k, A}(\phi(., u)) .
$$

Note that since $\phi(., u): x \mapsto \phi_{1}(x) \phi_{2}(u(x))$ is bounded, by Proposition 5.1, $G_{k, A}(\phi(., u)) \in$ $\mathcal{C}_{0}(A)$ and then $T$ is well defined. Moreover, as $\phi_{2}$ is nondecreasing, for every $u \in \mathcal{M}$ and every $x \in \bar{A}$, we have

$$
c_{1} \leq P_{k, A}[f](x)-G_{k, A}\left[\phi\left(., c_{2}\right)\right](x) \leq T(u)(x) \leq P_{k, A}[f](x) \leq c_{2} .
$$

Hence, we have $T(\mathcal{M}) \subset \mathcal{M}$.

Now, we want to establish that $T$ has a unique fixed point in $\mathcal{M}$ by using the Schauder theorem.

- Firstly, we will prove that $T(\mathcal{M})$ is relatively compact. For this, we will use the ArzelàAscoli theorem. From (5.14), $T(\mathcal{M})$ is pointwise bounded.

Let $x_{0} \in A$. For every $u \in \mathcal{M}$ we have

$$
\begin{aligned}
\left|T(u)(x)-T(u)\left(x_{0}\right)\right| & \leq\left|P_{k, A}[f](x)-P_{k, A}[f]\left(x_{0}\right)\right|+\left|G_{k, A}[\phi(., u)](x)-G_{k, A}[\phi(., u)]\left(x_{0}\right)\right| \\
& \leq\left|P_{k, A}[f](x)-P_{k, A}[f]\left(x_{0}\right)\right| \\
& +\int_{A}\left|G_{k, A}(x, y)-G_{k, A}\left(x_{0}, y\right)\right| \phi_{1}(y) \phi_{2}(u(y)) \omega_{k}(y) d y \\
& \leq\left|P_{k, A}[f](x)-P_{k, A}[f]\left(x_{0}\right)\right| \\
& +\phi_{2}\left(c_{2}\right)\left\|\phi_{1}\right\|_{\infty} \int_{A}\left|G_{k, A}(x, y)-G_{k, A}\left(x_{0}, y\right)\right| \omega_{k}(y) d y .
\end{aligned}
$$

Therefore, from (5.13) and the continuity of the function $P_{k, A}[f]$, we conclude that $T(\mathcal{M})$ is equicontinuous. Finally, $T(\mathcal{M})$ is relatively compact as desired.

- Secondly, we will prove that $T: \mathcal{M} \longrightarrow \mathcal{M}$ is continuous. Let then $\left(u_{n}\right)$ be sequence in $\mathcal{M}$ which converges uniformly to $u \in \mathcal{M}$. We have

$$
\left|T\left(u_{n}\right)(x)-T(u)(x)\right| \leq \int_{A} G_{k, A}(x, y) \phi_{1}(y)\left|\phi_{2}\left(u_{n}(y)\right)-\phi_{2}(u(y))\right| \omega_{k}(y) d y .
$$

But

$$
0 \leq G_{k, A}(x, y) \phi_{1}(y)\left|\phi_{2}\left(u_{n}(y)\right)-\phi_{2}(u(y))\right| \leq 2 \phi_{2}\left(c_{2}\right)\left\|\phi_{1}\right\|_{\infty} G_{k, A}(x, y) .
$$


Thus, we can use the dominated convergence theorem to obtain that $T\left(u_{n}\right) \longrightarrow T(u)$ pointwise. Hence, by equicontinuity, we get the uniform convergence.

Consequently, there exists $u \in \mathcal{M}$ such that

$$
u=T(u)=P_{k, A}[f]-G_{k, A}(\phi(., u)) .
$$

Finally, note that from the properties of $P_{k, A}$ as well as (5.10), $u$ is a solution of (5.4). This finishes the proof of the theorem.

\section{References}

[1] S. Axler, P. Bourdon and W. Ramey. Harmonic Function Theory. Springer Verlag, Second edition (2001).

[2] M. Ben Chrouda. On the Dirichlet problem associated with the Dunkl Laplacian. Ann. Polon. Math.117(1), (2016), 79-87.

[3] M. Ben Chrouda, K. El Mabrouk and K. Hassine. Boundary value problem for the Dunkl Laplacian. Accepted in the Journal of Prob. and Math. Stat.

[4] J. F. van Diejen and L. Vinet. Calogero-Sutherland-Moser Models. Springer-Verlag, CRM Series in Mathematical Physics (2000).

[5] F.Dai and Y. Xu. Approximation Theory and Harmonic Analysis on Spheres and Balls. Springer, (2013).

[6] C. F. Dunkl. Differential-difference operators associated to reflection groups. Trans. Amer. Math. Soc., 311, (1989), 167-183.

[7] C. F. Dunkl. Integral kernels with reflection group invariance. Canad. J. Math., 43, (1991), 123-183.

[8] C. F. Dunkl and Y. Xu. Orthogonal Polynomials of Several variables. Cambridge Univ. Press (2001).

[9] J. El Kamel and CH. Yacoub. Poisson integrals and Kelvin transform associated to Dunkl-Laplacian operator. Global Journal of Pure and Applied Math. (2007), Vol. 3, Issue 5, p. 351.

[10] P. Etingof. Calogero Moser systems and representation theory. Zürich Lectures in Advanced Mathematics, European Mathematical Society (EMS), Zürich, (2007).

[11] L. Gallardo and C. Rejeb. A new mean value property for harmonic functions relative to the Dunkl-Laplacian operator and applications. Trans. Amer. Math. Soc., Vol. 368, Number 5, May 2016, p.3727-3753.

[12] L. Gallardo and C. Rejeb. Radial mollifiers, mean value operators and harmonic functions in Dunkl theory. J. Math. Anal. Appl. (2017), Volume 447, Issue 2, 1142-1162.

[13] L. Gallardo and C. Rejeb. Newtonian Potentials and subharmonic functions associated to root systems. Journal of Potential Analysis, 47 (2017), 369-400.

[14] L. Gallardo, C. Rejeb and M. Sifi. Riesz potentials of Radon measures associated to reflection groups. Accepetd in Adv. in Pure and Applied Math. https://doi.org/10.1515/apam-2017-0057.

[15] P. Graczyk, T. Luks and M. Rösler. On the Green Function and Poisson Integrals of the Dunkl Laplacian. Journal of Potential Analysis, Volume 48, Issue 3 (2018), 337-360.

[16] M. Grossi and D. Vujadinović. On the Green Function of the Annulus. Anal. Theory Appl., Vol. 32, No. 1 (2016), 52-64.

[17] K. Hassine. Mean value property of $\Delta_{k}$-harmonic functions on $W$-invariant open sets. Afr. Mat. 27(7), (2016), 1275-1286.

[18] J. E. Humphreys. Reflection groups and Coxeter groups. Cambridge Studies in Advanced Mathematics 29, Cambridge University Press, (1990). 
[19] M. Maslouhi and E. H. Youssfi. Harmonic functions associated to Dunkl operators. Monatsh. Math. 152 (2007), 337-345.

[20] H. Mejjaoli and K. Trimèche. On a mean value property associated with the Dunkl Laplacian operator and applications. Integ. Transf. and Spec. Funct., 12(3), (2001), 279-302.

[21] M. Rösler. Generalized Hermite polynomials and the heat equation for Dunkl operators. Comm. Math. Phys, 192, (1998), 519-542.

[22] M. Rösler and M. Voit. Markov processes related with Dunkl operators. Adv. in Appl. Math. 21 (1998), 575-643.

[23] M. Rösler. Positivity of Dunkl's intertwining operator. Duke Math. J., 98, (1999), 445-463.

[24] M. Rösler. Dunkl Operators: Theory and Applications. Lecture Notes in Math., vol.1817, Springer Verlag (2003), 93-136.

[25] G. Szegö. Orthogonal Polynomials. Amer. Math. Soc., Providence, RI, Fourth edition, (1975).

[26] K. Trimèche. The Dunkl intertwining operator on spaces of functions and distributions and integral representation of its dual. Integ. Transf. and Spec. Funct., 12(4), (2001), 394-374.

[27] Y. Xu. Integration of the intertwining operator for h-harmonic polynomials associated to reflection groups. Proc. Amer. Math. Soc. 125 (1997), 2963-2973. 